\title{
Landscape configuration drives persistent spatial patterns of occupant distributions
}

\author{
Elizabeth A. Hamman ${ }^{1}$ (D) . Scott A. McKinley ${ }^{2}$ Adrian C. Stier ${ }^{3}$. \\ Craig W. Osenberg ${ }^{1}$
}

Received: 1 May 2017 / Accepted: 23 October 2017

(c) Springer Science+Business Media B.V. 2017

\begin{abstract}
Variation in the density of organisms among habitat patches is often attributed to variation in inherent patch properties. For example, higher quality patches might have higher densities because they attract more colonists or confer better post-colonization survival. However, variation in occupant density can also be driven by landscape configuration if neighboring patches draw potential colonists away from the focal habitat (a phenomenon we call propagule redirection). Here, we develop and analyze a stochastic model to quantify the role of landscape configuration and propagule redirection on occupant density patterns. We model a system with a dispersive larval stage and a sedentary adult stage. The model includes sensing and decisionmaking in the colonization stage and density-dependent mortality (a proxy for patch quality) in the post-colonization stage. We demonstrate that spatial variation in colonization is retained when the supply of colonists is not too high, postcolonization density-dependent survival is not too strong,
\end{abstract}

Electronic supplementary material The online version of this article (https://doi.org/10.1007/s12080-017-0352-1) contains supplementary material, which is available to authorized users.

Elizabeth A. Hamman

hamman@uga.edu

1 Odum School of Ecology, University of Georgia, 140 E. Green St. Athens, GA 30602-2202, USA

2 Department of Mathematics, Tulane University, 6823 St. Charles Ave New Orleans, LA 70118, USA

3 Department of Ecology Evolution and Marine Biology, University of California Santa Barbara, Santa Barbara, CA 93106-9620, USA and colonization events are not too frequent. Using a reef fish system, we show that the spatial variation produced by propagule redirection is comparable to spatial variation expected when patch quality varies. Thus, variation in density arising from the spatial patterning of otherwise identical habitat can play an important role in shaping long-term spatial patterns of organisms occupying patchy habitats. Propagule redirection is a potentially powerful mechanism by which landscape configuration can drive variation in occupant densities, and may therefore offer new insights into how populations may shift as landscapes change in response to natural and anthropogenic forces.

Keywords Propagule redirection - Settlement shadows . Density dependence $\cdot$ Patch selection

\section{Introduction}

Many landscapes are fragmented, either naturally or through human activity, yielding a system of patches heterogeneous in size and connectivity. Accordingly, the distribution of organisms that occupy these patches is also often heterogeneous, with high densities of organisms in some patches, but low densities in others. Differences in the density of organisms among patches are often attributed to differences in the intrinsic properties of a patch that affect the colonization of the patch or survival within the patch. For example, organisms differentially colonize patches based on size (Sih and Baltus 1987), quality (Tolimieri 1995; Holbrook et al. 2000), micro-environmental conditions (Lecchini et al. 2003), the presence of predators (Wesner et al. 2012), the presence of conspecifics (Stamps 1988), and the presence of heterospecifics (Mönkkönen et al. 1990). Properties extrinsic to the patch, such as the surrounding habitat matrix (e.g., 
Gustafson and Gardner (1996) and Ricketts (2001)) or the spatial configuration of patches (e.g., MacArthur and Wilson (1967) and Stier and Osenberg (2010)) also lead to variation in density through their effects on colonization. In such cases, intrinsically identical patches may harbor very different densities of organisms (e.g., Stier and Osenberg (2010)).

The population-level effects of differential patterns of colonization of patches within a network is well articulated in the metapopulation and metacommunity literature (e.g., Hanski (1994), Gonzalez et al. (1998), and Koelle and Vandermeer (2005)). In these studies, neighboring patches increase colonization (e.g., Hill et al. (1996) and Eaton et al. (2014)) by providing propagules. However, this literature has rarely examined how otherwise hospitable neighboring patches might reduce colonization.

In contrast to the metapopulation literature, the foraging literature has explored possible positive and negative effects of patch density and configuration (e.g., Ryall and Fahrig (2006)). For example, if a plant (i.e., "patch") has few flowers, it may not attract pollinators. But, if that plant is near another, then the combined floral display may recruit pollinators into the general area, increasing the visitation rate of pollinators to plants and individual flowers (reviewed in Mitchell et al. (2009)). However, plants with many neighbors may have decreased per-capita visitation rates from pollinators because flowers compete with one another for the potentially limited supply of pollinators (see review by Morales and Traveset (2008)). Similar beneficial and deleterious effects of patch density observed in these behavioral studies may also exist at the population level. Thus, models of spatial population dynamics that incorporate potential deleterious effects of neighboring patches might inform our understanding of the dynamics of organisms that occupy heterogeneous landscapes.

Metapopulations and foraging (e.g., pollinator) systems form a dichotomy with respect to the scale of migration and patch size. In metapopulations, individuals can be born, mature, and reproduce within a single patch; migration rates among metapopulations also are typically low. In contrast, foragers must visit multiple patches within a short time frame-no single patch is large enough to support the dynamics of an entire population. Many systems are intermediate with respect to these extremes.

For example, adult aquatic beetles colonize ponds (after emerging from terrestrial pupation sites) where they reproduce and frequently remain for the rest of their life. Colonization rates in one pond (a patch) could be influenced by its own characteristics as well as the landscape of ponds in which the focal pond occurs. Experimental tests (Resetarits and Binckley 2009, 2013) demonstrated that beetle colonization was reduced in ponds that contained predators, demonstrating an effect of patch quality. However, colonization also was reduced in predator-free ponds if they occurred near ponds that contained predators, showing that variation in quality of neighboring ponds also affected colonization. The experiments conducted by Resetarits and Binckley also manipulated the total number of ponds at a site. In contrast to the results for pond quality, they did not find any discernible effect of patch number on per pond colonization. Thus, in this system, it was the quality of the patch or the landscape (i.e., in terms of predator density) and not simply the availability of ponds (i.e., landscape configuration) that drove colonization patterns

In contrast, Stier and Osenberg (2010) varied landscape configuration of corals (keeping intrinsic properties of corals the same) and found that the presence of neighboring corals reduced the per-coral colonization rates of focal coral patches by larval reef fish by over $70 \%$. Although there was an increase in total colonization with increased habitat, it was not proportional to the total amount of habitat. Thus, neighboring patches (i.e., corals) redirected colonists from focal patches, resulting in propagule redirection (also see Carr and Hixon (1997), Osenberg et al. (2002a), and Morton and Shima (2013)). As a result of this "competition" among neighboring patches for colonists, the addition of new patches to a region may limit the potential increase in total colonization. At the extreme, there may be no increase at the regional level. These two examples (beetles and fishes) illustrate how the strength of propagule redirection can vary across systems. Reef fish represent one point near the end of this redirection spectrum, in which strong redirection results in spatial heterogeneity in colonization due to the presence or absence of neighboring patches (Stier and Osenberg 2010), whereas aquatic beetles represent the other end of the spectrum, in which weak redirection (neighboring ponds do not affect colonization patterns) leads to colonization patterns that are independent of the local landscape configuration.

Variable colonization driven by the combined effects of the spatial distribution of (otherwise identical) habitat patches and propagule redirection can have long-term consequences for population dynamics (Stier and Osenberg 2010), especially for species in which colonists remain associated with habitat patches for most of their life history (as is the case for the beetles and reef fish). While the strength of propagule redirection (and other processes that affect colonization) establishes the initial spatial pattern of colonists, the degree to which these patterns propagate over longer time frames will depend on the post-colonization processes. These post-colonization processes will be affected by within-patch factors such as the local environment and density dependence. For example, mortality of newly settled fish is often density-dependent (Osenberg et al. 2002b; Hixon and Jones 2005; White et al. 2010), with negative density dependence possibly arising through competition 
for refuges from predators on the interior of the coral colony (e.g., Holbrook and Schmitt (2002)). When densitydependent mortality is strong, the spatial variation in colonization resulting from propagule redirection may be eliminated as large cohorts are quickly and disproportionately reduced in density relative to patches with few colonists. In contrast, when density-dependent mortality is weak, we expect the spatial patterns established during colonization to persist through the post-colonization phase.

Here, we develop a model to investigate how spatial patterns can be created in a landscape consisting of identical habitat patches. In particular, we investigate how propagule redirection leads to different long-term spatial patterns of occupants that depend on the proximity of neighboring patches. We also consider how density-dependent mortality, density-independent mortality, colonist supply, and colonization frequency affect the long-term spatial patterns that result from different forms of propagule redirection. Finally, we apply our framework to a reef fish system, using data to parametrize our model. Because our model assumes homogeneous quality, we also contrast predictions derived from our propagule redirection model to one including variation in habitat quality.

\section{Methods}

Although our approach is general, we develop it with reference to corals (habitat patches) and coral occupants (such as fish). This is a demographically open system where colonists to a patch are not the progeny of the local adults. In most coral reef systems, late-stage fish larvae (produced by relatively distant adults, but see Jones et al. (2009)) enter shallow reef areas from the pelagic zone and settle from the water column into a coral. After settlement (i.e., colonization), many coral occupants have limited ability to move to other corals due to factors such as predation (Holbrook and Schmitt 1997, 2002). Thus, the occupants often reside within the colonized coral for the entire juvenile and adult portions of their life cycle. Extrapolation to other contexts can be done by slightly redefining life stages. For example in the beetle example, adults, rather than larvae, colonize patches.

Our model includes three components, each of which corresponds to sequential events that start with a larval fish's arrival on the reef and end with its death: (1) potential settlers (i.e., colonists) arrive at random locations uniformly distributed in the landscape, (2) potential settlers choose a coral based on relative signals received from corals in the landscape, and (3) settlers that successfully arrive at a coral survive (or die) over the inter-pulse interval. The first two phases collectively define the "settlement phase," while the third defines the "post-settlement phase." We assumed settlement events are discrete, pulsed events and occur at regular time intervals, mirroring the life history of many marine organisms, while survival occurs continuously in time (see Fig. 7 in Appendix B). Corals in the landscape are identical in all ways (e.g., size and quality), except for their spatial proximity to other corals. The area surrounding the corals also is assumed uniform.

As a result, spatial variation in expected patch occupancy is only driven by the effects of landscape configuration (i.e., the number of nearby patches) on settlement. However, some additional variation arises because each model component is intrinsically stochastic, leading to variation in the spatial arrangement of corals, larval density, and the survival of fish. Therefore, we developed a stochastic model of settlement and post-settlement dynamics on a spatially explicit landscape. We also developed a deterministic approximation to the stochastic model to gain an understanding of the role of specific processes. We start with a general description of the model.

\section{Settlement processes}

Settlement (colonization) events occur in a landscape of identical, discrete, circular habitat patches (corals). Settlers (larval fish), arrive in the landscape, perceive signals from coral patches, and choose a coral based upon these signals (e.g., Lecchini et al. (2005) and Dixson et al. (2014)). Potential settlers then travel to the selected coral but incur mortality in transit. Because reef fish settlement events are pulsed and often linked with the lunar cycle (e.g., Robertson (1992)), we model settlement events discretely in time.

For a settlement event, we assume that the density of arriving larvae is $L$, and the initial locations of the larvae are independent and randomly distributed over the landscape: i.e., the distribution of potential settlers is a spatial Poisson point process with density $L$. The number of settling fish larvae in the landscape then has the distribution $S \sim \operatorname{Pois}(L|\mathcal{O}|)$, where $\mathcal{O}$ is our notation for the landscape, and $|\mathcal{O}|$ is its area.

Let $\left\{\left(x_{i}, y_{i}\right)\right\}_{i=1}^{K}$ be the locations of $K$ corals in the landscape. Each coral has radius $R$, signal magnitude $m$, and signal degradation rate $\rho$. On the coral, the signal has strength $m$, but as distance from the coral increases, we assume the strength of the signal decreases exponentially. Therefore, a potential settler arriving at location $(x, y)$ perceives a signal from coral $i$ of magnitude

$\Lambda_{i}(x, y):=m e^{-\rho d_{i}(x, y)}$

where $d_{i}(x, y)$ is the distance from the potential settler's location $(x, y)$ to the edge of coral $i$ (the center of the coral is located at $\left.\left(x_{i}, y_{i}\right)\right)$ :

$d_{i}(x, y):=\max \left(0, \sqrt{\left(x-x_{i}\right)^{2}+\left(y-y_{i}\right)^{2}}-R\right)$. 
A settler selects a coral with a probability that is equal to the relative strength of the signals: a settler arriving at position $(x, y)$ will choose coral $i$ with probability $\Lambda_{i}(x, y) /\left(\sum_{j} \Lambda_{j}(x, y)\right)$, so long as the total signal strength exceeds a detection threshold, $\theta$. If the total signal is less than $\theta$, the settler dies. Once a settler chooses a coral, it must survive the travel to the selected coral. We assume that the mortality rate and velocity during travel is constant, so that the probability of survival starting at location $(x, y)$ and traveling to coral $i$ is $e^{-\mu d_{i}(x, y)}$.

To obtain the settlement to a focal coral, we determine $\lambda_{i}$, the number of settlers that select and survive the travel to coral $i$. If there are $S$ settlers in the entire landscapewide cohort, then $\lambda_{i}$ is a Binomial random variable where $S$ is the number of "trials," and $p_{i}$ is the "success probability" that, given a settler detects enough signal to choose a coral, a given settler will choose, and survive travel to, coral $i$ :

$p_{i}=\frac{1}{|\mathcal{O}|} \iint_{\mathcal{O}} \frac{\Lambda_{i}(x, y)}{\sum_{j} \Lambda_{j}(x, y)} e^{-\mu d_{i}(x, y)} d x d y$.

Because we assume that the settler choices and survival are independent of each other, the number of arrivals at the focal coral is a thinned Poisson random variable, $\lambda_{i} \sim$ $\operatorname{Pois}\left(L|\mathcal{O}| p_{i}\right)$. In subsequent sections, when referring to the parameters of a focal coral, we will suppress the dependence on the index $i$ in the notation. Thus, a coral with $n$ residents transitions to having $n+\lambda$ residents when $t=\gamma k$ where $\gamma$ is the time between settlement pulses assumed to occur on a lunar cycle of 28 days (Table 1) and $k$ is an index that keeps track of subsequent cohorts.

\section{Post-settlement processes}

In contrast to the periodic structure of the settlement pulse events, post-settlement deaths occur on each coral continuously in time due to density-independent and density-dependent mortality. We used a stochastic version of a framework previously used successfully to model reef fish recruitment dynamics (Osenberg et al. 2002b; Shima and Osenberg 2003). The number of residents or fish in a coral patch at a time $t$, denoted $N(t)$, decreases as fish die due to densityindependent mortality $\alpha$ and density-dependent mortality $\beta$. We assume that mortality rates and density-dependent effects are independent of fish age and thus aggregate all cohorts together into a single measure of density, $N$.

Because many corals have very low numbers of residents and therefore demographic stochasticity has a potentially large effect, we used a continuous-time Markov chain. To simulate mortality, we used Gillespie's method (Gillespie 1977). If a coral has $n$ occupants, the time until the next one dies (i.e., the coral has $n-1$ occupants) is an exponential random variable with rate parameter $\alpha n+\beta n^{2}$, which captures the effects of density-independent and densitydependent factors on the loss rate (Osenberg et al. 2002b; Shima and Osenberg 2003). We simulated dynamics for the amount of time between settlement events (Table 1), and when populations are large, this discrete process is well approximated by a continuous dynamic (see Fig. 6 in Appendix A).
Table 1 List of parameters and dimensionless groups.

\begin{tabular}{|c|c|c|}
\hline \multicolumn{3}{|l|}{ Parameters } \\
\hline Description & Label & Values used for stochastic simulations \\
\hline Coral radius & $R$ & $1 \mathrm{~m}$ \\
\hline Coral signal magnitude & $m$ & 1 \\
\hline Coral signal decay rate ${ }^{a}$ & $\rho$ & $0.75 \mathrm{~m}^{-1}$ \\
\hline Coral signal detection threshold ${ }^{\mathrm{a}}$ & $\theta$ & 0.0001 \\
\hline Larvae survival decay rate ${ }^{\mathrm{a}}$ & $\mu$ & $0.4 \mathrm{~m}^{-1}$ \\
\hline Density-independent mortality rate ${ }^{b}$ & $\alpha$ & $0.0001 \mathrm{day}^{-1}$ \\
\hline Time between settlement events ${ }^{\mathrm{d}}$ & $\gamma$ & 28 days \\
\hline Density-dependent mortality rate ${ }^{\mathrm{b}, \mathrm{c}}$ & $\beta$ & 0.00056 and $0.056 \mathrm{~m}^{2} \mathrm{fish}^{-1} \mathrm{day}^{-1}$ \\
\hline Larval density $^{\mathrm{a}}$ & $L$ & 0.33 larvae $\mathrm{m}^{-2}$ \\
\hline \multicolumn{3}{|l|}{ Dimensionless groups } \\
\hline Description & Label & Definition \\
\hline $\begin{array}{l}\text { Relative strength of density-dependent } \\
\text { processes to density-independent pro- } \\
\text { cesses }\end{array}$ & $v$ & $\frac{\beta \bar{\lambda}}{\alpha}$ \\
\hline $\begin{array}{l}\text { Time between settlement events } \\
\text { relative to occupant longevity }\end{array}$ & $\Delta$ & $\alpha \gamma$ \\
\hline
\end{tabular}

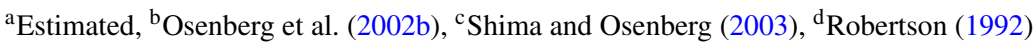




\section{Analysis and results}

We took two complementary approaches to analyze and interpret the stochastic, spatially explicit model and evaluate the effects of propagule redirection under different landscape configurations. First, we simulated both settlement and post-settlement dynamics using the parameters described above. Second, we constructed a deterministic approximation of the stochastic model and explored a nondimensionalized parameter space. Below, we explain each approach and provide the results of our analyses. Finally, we explore the ramifications of propagule redirection in a realistic system and compare the effects of propagule redirection to variation in habitat quality.

\section{Simulations of the full stochastic model}

\section{Settlement processes}

To determine how spatial patterns might be produced (or diminished) from spatial variation in settlement arising from propagule redirection within a heterogeneous landscape, we implemented a fully stochastic version of the model and manipulated the landscape configuration. We used three types of landscapes: landscapes with evenly distributed patches, randomly distributed patches, and clustered patches. We created evenly distributed landscapes
(Fig. 1a) using Spatstat's simple sequential inhibition model (Baddeley and Turner 2005), random landscapes (Fig. 1b) using a spatial Poisson process, and clustered landscapes (Fig. 1c) using a preferential attachment model (Hein and McKinley 2013).

For each landscape type, we created 100 replicate landscapes each with 40 corals for each landscape configuration. For each replicate landscape, we simulated settlement and post-settlement dynamics using the parameters in Table 1 . For each of the $3 \times 100$ runs, we simulated dynamics for at least 50 settlement events (the actual number was randomly selected to fall between 50 and 150 to avoid subtle cycles in population size). This was sufficient time for the coral to come to equilibrium (for an example, see Fig. 7 in Appendix B) and corresponds to a time scale for 4-12 years given that one settlement pulse occurs every lunar month. We recorded the mean number of settlers on each coral and the number of occupants on each coral just before the next settlement event would have occurred (our qualitative results are unaffected by the timing of sampling relative to the settlement pulse). To reduce edge effects, we only analyzed the interior by creating a buffer of $10 \%$ of the landscape width on all sides.

The three landscapes (Fig. 1a-c) resulted in different mean settlement rates. The clustered landscape (Fig. 1c) had a lower mean settlement rate (Fig. 1f) compared to the more evenly distributed landscapes (Fig. 1a, b) because fewer fish
Fig. 1 Landscape variation leads to heterogeneity in occupancy patterns. The top row of images (panels $\mathbf{a}, \mathbf{b}$ and $\mathbf{c}$ ) show the three sample landscapes, each corresponding to one landscape type (even, random, and clustered). In the middle row (panels $\mathbf{d}, \mathbf{e}$, and $\mathbf{f}$ ), we show the result of running a single settlement event, and the bottom row (panels $\mathbf{g}, \mathbf{h}$, and $\mathbf{i}$ ) the equilibrium occupancy for a single simulation. We emphasize the connection between the histograms and their associated landscapes through color coding: the gradient of isolation from blue for the most clustered coral and red for the most isolated coral is indicated in all images. $\mu=0.4, \rho=0.75$, $\alpha=0.0001, \beta=0.00056$, $\gamma=28, R=1$, and $L=0.33$ (a)
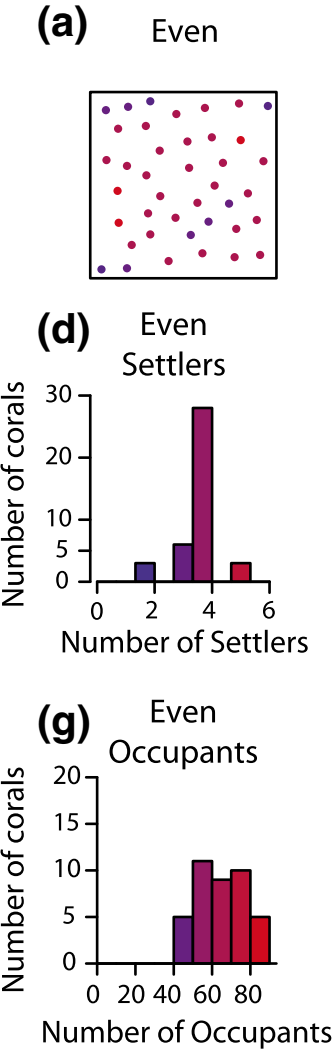
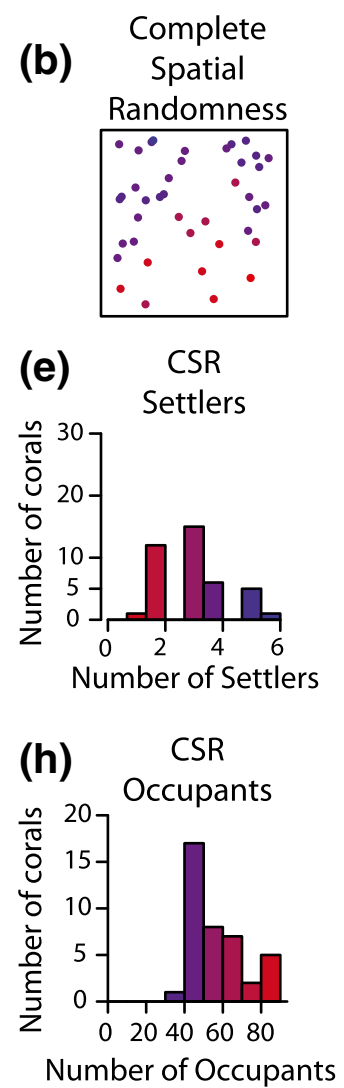

(c) Clustered

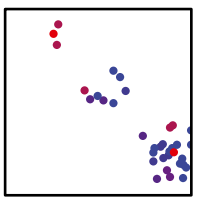

(f)

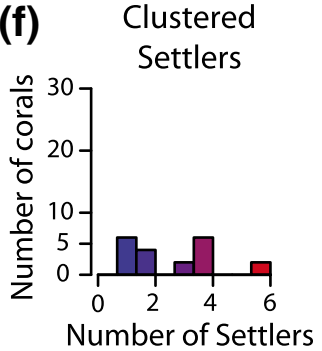

(i)

Clustered

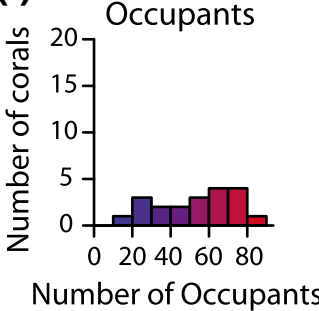


were able to survive the transit to a coral when the corals were clustered: i.e., the average distance to a coral was greater in the clustered landscape than the even landscape. The three landscapes (Fig. 1a-c) differed in the resulting spatial variation in settlement. Landscapes with evenly distributed patches (Fig. 1a, d) had very little variation in settlement because all corals had similar neighborhoods and thus similar degrees of propagule redirection. In contrast, corals in clustered landscapes had the greatest amount of variation in settlement (Fig. 1f) because some corals were surrounded by other corals on all sides, whereas some corals had relatively few neighbors (i.e., compare the red vs. blue points in Fig. 1c, f). Landscapes with complete spatial randomness were intermediate in variance. Across all of the simulated landscapes, the maximum and minimum settlement rates varied 2.9 \pm 0.04 SE-fold, $3.4 \pm 0.06 \mathrm{SE}-$ fold, and $4.7 \pm 0.11 \mathrm{SE}$-fold for the landscapes with evenly distributed, randomly distributed, and clustered patches, respectively.

\section{Post-settlement processes}

This heterogeneity in settlement resulted in spatial variation in the long-term number of occupants (Fig. 1g, f, i): i.e., the density of occupants was more heterogeneous in the clustered landscapes (compare Fig. 1g with Fig. 1i). The results, depicted in Fig. 1, arose for one particular set of landscape configurations and parameter values. We therefore conducted the simulations for two levels of density dependence, moderate and strong $(\beta=0.056$ and 0.00056 : see below, "Application to a realistic system") and summarized the results in each landscape using an index of dispersion (variance/mean) for settlers and occupants. The index of dispersion for larvae was always one, by definition (indicating a random distribution, Fig. 2a).

Although larvae were randomly distributed, the spatial dispersion of settlers was more heterogeneous than a Poisson spatial process in all landscapes due to propagule redirection. The greatest heterogeneity occurred in the clustered landscapes (Fig. 2b, also seen in Fig. 1). Because settlement was unaffected by post-settlement processes, dispersion of settlers did not vary across the two levels of density dependence. The translation of this spatial heterogeneity in settlement to long-term spatial variation in occupant density depended upon the strength of densitydependent mortality (Fig. 2c). When density dependence was moderate $(\beta=0.00056)$, there was demonstrable variation in density in both the random and clustered landscape configurations, although heterogeneity was the greatest in the landscapes with clustered corals, and when corals were evenly distributed, the little heterogeneity established during settlement faded due to the small amount of
Fig. 2 Spatial heterogeneity in a larvae, $\mathbf{b}$ settlers, and $\mathbf{c}$ occupants for three landscape types (even, random, and clustered). An index of dispersion (variance/mean) $>1$ indicates a heterogeneous distribution for the focal life stage, $=1$ indicates a random (dashed horizontal line), and $<1$ indicates a more even distribution. For each landscape type, we simulated 100 replicate landscapes, each with 40 corals. Results are given for the end of the simulation (i.e., after at least 50 settlement pulses). In all simulations, we set $\mu=0.4$, $\rho=0.75, \alpha=0.0001, \gamma=28$, $R=1, L=0.33$, and $\beta=0.00056$ (moderate) and $\beta=0.056$ (strong). Error bars represent $95 \%$ confidence intervals (based on the 100 landscapes)
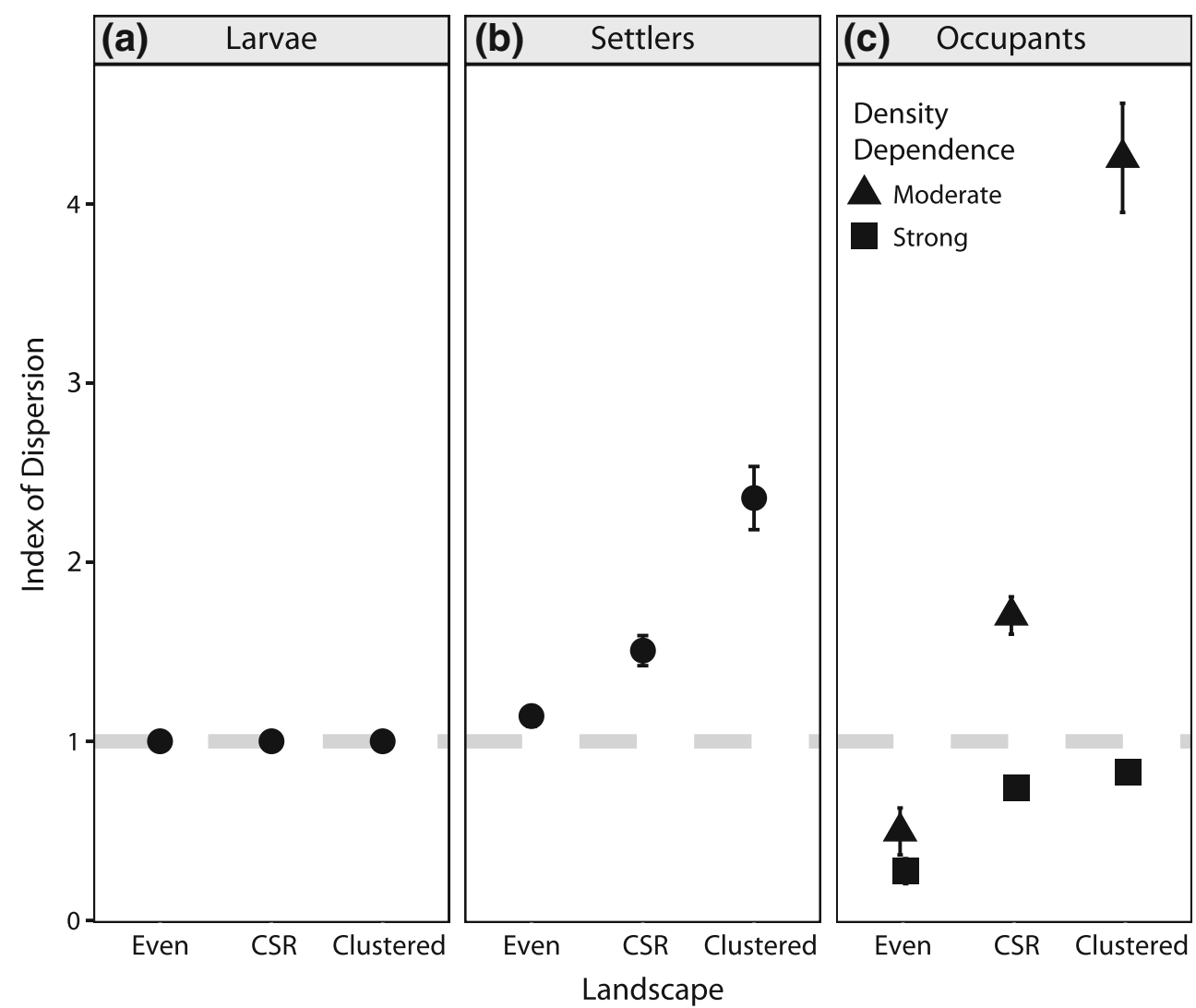
density-dependent mortality in post-settlement dynamics (Fig. 2c). This pattern mirrored that seen in the settlement stage (compare Fig. 2b, c), although the values were larger due to the buildup of multiple cohorts. In contrast, when density dependence was strong $(\beta=0.056)$, spatial variation was eliminated, even when settlement was highly heterogeneous (Fig. 2c). At the most intense levels of density dependence, the index of dispersion was $<1$, indicating that densities were more homogeneous than expected given uniformly random larval rain.

In summary, these simulations show that spatial heterogeneity in occupants can be created in a landscape of otherwise identical habitat patches, if (1) there is propagule redirection (i.e., habitat competes for colonists: Stier and Osenberg (2010)), (2) the distribution of patches in the landscape creates spatial variation in patch configurations (Figs. 1 and 2), and (3) post-settlement density dependence is sufficiently weak that the variation in settlement persists over the long term (Figs. 1 and 2).

\section{Analytic approximation}

Because there is no analytic solution for certain long-term properties of the above stochastic model, we analyzed a deterministic approximation. Here, we introduce the analytic approximation and a simple case of a clustered coral and an isolated coral to explore the relationship between the model parameters and long-term patterns. For a focal coral, we used the ODE to model $\bar{N}$, or the average number of occupants on a coral,

$\frac{d \bar{N}}{d t}=-\alpha \bar{N}-\beta \bar{N}^{2}+\sum_{k=0}^{\infty} \bar{\lambda} \delta_{\gamma k}(t), \quad \bar{N}(0)=N_{0}$,

where the parameters $\alpha$ (density-independent mortality rate), $\beta$ (density-dependent mortality rate), and $\bar{\lambda}$ (average number of settlers per settlement event) are the same as above. In the last term, we use a sequence of Dirac-delta functions to produce instantaneous pulses at the times $\gamma k$ of average size $\bar{\lambda}$.

For reasonably large population sizes, this ODE closely approximates the stochastic system (Appendix A in the Supporting Information). Studying the long-term behavior of $\bar{N}(t)$ provided valuable insights that facilitated our interpretations of the simulation results and the behavior of the system in various regions of the parameter space described by the density-independent mortality rate $(\alpha)$, density-dependent mortality rate $(\beta)$, time between arrivals $(\gamma)$, and the average number of settlers during a settlement event $(\bar{\lambda})$.

To simplify the analysis, we calculated the number of settlers on a focal coral in two extreme habitat configurations: (a) when a coral is completely isolated (i.e., when settlement is maximized), $\bar{\lambda}_{\text {isolated }}$, and (b) when a coral is completely surrounded by other corals (i.e., when settlement is minimized), $\bar{\lambda}_{\text {clustered. }}$ The expected number of settlers to an isolated coral and to a clustered is (Appendix $\mathrm{C}$ ) as follows:

$$
\begin{aligned}
\bar{\lambda}_{\text {isolated }} & =L \pi R^{2}+\frac{2 \pi L}{\mu^{2}}\left[\mu R+1-\left(\frac{m}{\theta}\right)^{-\mu / \rho}\left(\frac{\mu}{\rho} \ln \left(\frac{m}{\theta}\right)\right.\right. \\
& +\mu R+1)] \\
\bar{\lambda}_{\text {clustered }} & =L \pi R^{2} .
\end{aligned}
$$

The number of settlers to a clustered coral is simply the larvae that arrive directly over the patch, while an isolated coral receives those that land directly over the coral as well as those that arrive within the detection region and survive the travel to the coral. The difference in settlement is driven by the second term in Eq. 5a, which provides the number of settlers that arise from larvae that must swim to the coral from other locations (and survive the journey). When mortality $(\mu)$ is low (or corals are small), the relative variation in the number of settlers (to an isolated vs. a clustered coral) is very high.

To study the approximation (Eq. 4), we reduced the complexity of the analysis via non-dimensionalization (Appendix D) by substituting $\tau=\alpha t$ and defining two dimensionless parameter groups,

$v:=\frac{\beta \bar{\lambda}}{\alpha}$ and $\Delta:=\alpha \gamma$.

The parameter $v$ is a measure of the relative strength of the density-dependent processes and is proportional to the number of settlers per settlement event and the ratio of density-dependent processes to density-independent mortality. The parameter $\Delta$ weighs the timing of the settlement events to the strength of density-independent mortality. We further simplified the equation by substituting $\tilde{N}=\frac{\bar{N}}{\alpha / \beta}$ and introducing a non-dimensional delta-function $\tilde{\delta}(\tau)=\frac{\delta(t)}{\alpha}$, we obtain

$\frac{d \tilde{N}}{d \tau}=-\tilde{N}(1+\tilde{N})+v \sum_{k=0}^{\infty} \tilde{\delta}_{k \Delta}(\tau)$

with $\tilde{N}(0)=\tilde{N}_{0}$.

To analyze the solution to Eq. 7, we took a two-step approach. First, we solved the system on intervals between settlement pulses (i.e., when only the post-settlement processes were acting). Specifically, suppose that the population is of size $n$ when the settlement pulse occurs $(t=k \Delta$ or one of the settlement peaks in Fig. 7). Then, during post-settlement phases, the solution takes the form

$\tilde{N}(\tau)=\frac{(n+v) e^{-(\tau-k \Delta)}}{1+(n+v)\left(1-e^{-(\tau-k \Delta)}\right)}$, for $\tau \in(k \Delta,(k+1) \Delta]$. 
Second, we found the equilibrium by setting the magnitude of settlement pulses equal to the deaths that occurred between each pulse. The equilibrium value of the population size immediately prior to a settlement event is (see Appendix B) as follows:

$$
\begin{aligned}
\tilde{N}^{*} & :=\lim _{k \rightarrow \infty} \tilde{N}(k \Delta) \\
& =\frac{1+v}{2}\left(-1+\sqrt{1+\frac{4 v e^{-\Delta}}{\left(1-e^{-\Delta}\right)(1+v)^{2}}}\right) .
\end{aligned}
$$

Equation 9 provides the equilibrium number of fish on a coral for a given level of settlement (reflected in $v$ ). That settlement is determined by the environment (which can affect larval supply and mortality during settlement) as well as the spatial configuration of the coral landscape (which affects the degree of propagule redirection). Thus, in a single landscape, there may be isolated corals for which settlement is high and other corals (i.e., those near other corals) for which settlement is low.

We used the cases of an isolated versus clustered coral (Eqs. 5a and 5b) to examine how the most extreme heterogeneity in settlement patterns is modified by post-settlement processes (as defined by $v$ and $\Delta$ ) to drive spatial patterns in the equilibrium number of occupants on the coral $\left(\tilde{N}^{*}\right)$. In particular, we determined to what extent and under what conditions the spatial variation in settlement is maintained as spatial variation in occupants. Long-term patterns

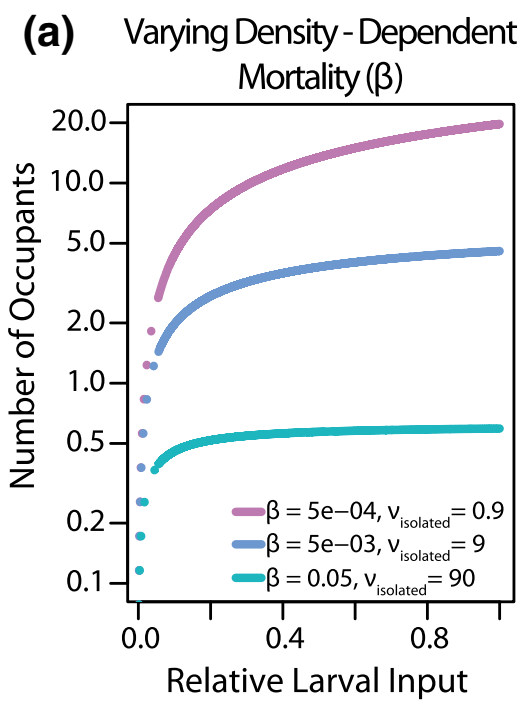

Fig. 3 The relationship between the equilibrium number of occupants and settlement rate for corals that vary in their degree of isolation (and thus their relative settlement) for three different levels of the non-dimensionalized parameter, $v$, achieved by changing either a the strength of density dependence, $\beta$, or b larval density $(L)$. Relative settlement gives the settlement to a coral relative to that received by an isolated coral. Thus, an isolated coral has a relative settlement of of occupant density (as reflected in Eq. 9) depend upon (1) settlement intensity (i.e., $\bar{\lambda}$, as reflected in $v$ ), (2) densitydependent mortality (i.e., $\beta$ as reflected in $v$ ), (3) densityindependent mortality (i.e., $\alpha$ as reflected in $v$ and $\Delta$ ), and (4) the time between settlement events (i.e., $\gamma$ as reflected in $\Delta$ ).

For $R=1$ and $\mu=0.4$, an isolated and a clustered coral would experience an almost 18 -fold difference in settlement: i.e., $\bar{\lambda}_{\text {isolated }} / \bar{\lambda}_{\text {clustered }}=17.9$. We then examined the conditions under which this disparity in settlement (i.e., as measured by $\bar{\lambda}_{\text {isolated }} / \bar{\lambda}_{\text {clustered }}=17.9$ ) led to disparity in the equilibrium number of occupants on corals (i.e., as measured by $\bar{N}_{\text {isolated }}^{*} / \bar{N}_{\text {clustered }}^{*}$ ). In particular, we examined this relationship under different values of $v$ obtained by changing either density dependence ( $\beta$, Fig. 3a), or larval supply (L, Fig. 3b).

As $v$ increases (i.e., the strength of density-dependent mortality and settlement intensity grows relative to the strength of density-independent mortality), the variation in the long-term number of occupants among corals (teal curves in Fig. 3a, b) decreases, despite the 17.9-fold variation in settlement: e.g., when $v=90$, the 17.9-fold variation in settlement was reduced to 1.5 -fold variation in equilibrium density (Fig. 3a, b). By contrast, as $v$ decreases, the variation in settlement rate persisted, creating longterm variation in occupancy patterns among patches (purple curves in Fig. 4): e.g., when $v=0.9$, the 17.9-fold variation settlement was reduced to only 7.3-fold (Fig. 3a, b).

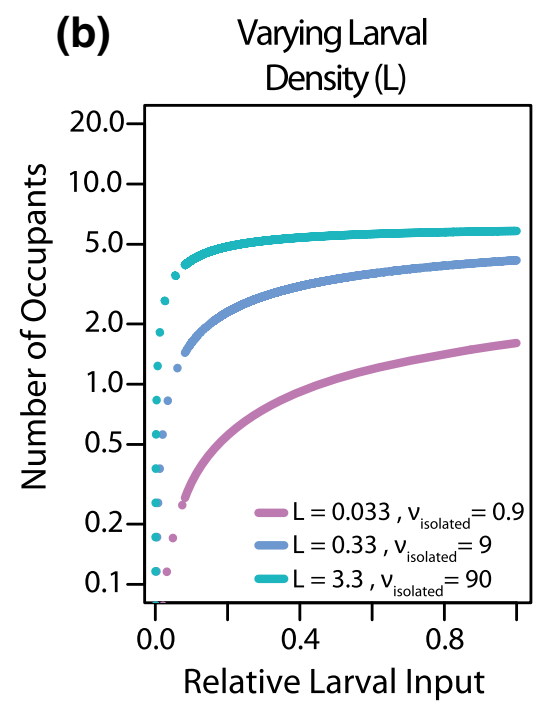

1.0. Solid lines indicate the equilbrium (Eq. 9) for corals with inputs between $\lambda_{\text {clustered }}$ and $\lambda_{\text {isolated. }}$. Dashed lines extend those relationships to settlement rates that are $<\lambda_{\text {clustered }}$ and thus reveal the full relationship. Colors indicate values of $v_{\text {isolated }}$ (the value for $v$ that corresponds to $v_{\text {isolated }}$, note that $\left.v_{\text {isolated }}=17.9 \times v_{\text {clustered }}\right): v_{\text {isolated }}=0.9$ (purple), $v_{\text {isolated }}=9$ (blue), and $v_{\text {isolated }}=90$ (teal). For these calculations, $R=1$ and $\mu=.4$, and $\Delta=0.28$ 
We also compared the relative equilibria on an isolated versus clustered coral across the full parameter space of the non-dimensionalized system (i.e., using Eq. 8). As $v \rightarrow 0$, the relative spatial variation in occupant density approached the relative variation in settlement (in this case, 17.9). When $v$ was small, (i.e., weak density dependence and low larval input), the heterogeneity in settlement persisted and led to long-term heterogeneity in the numbers of coral occupants. Thus, in undersaturated systems, longterm heterogeneity in the number of occupants can be driven solely by varying degrees of clustering among otherwise identical habitats. In contrast, increasing $v$ caused the system to become saturated, so differences in settlement due to propagule redirection mattered little to the equilibrium number of occupants on the coral: i.e., the equilbrium numbers on isolated and clustered corals were approximately equal (Fig. 4).

The effect of the time between settlement pulses (i.e., via $\Delta$ ) was more complicated. When $v$ was very small, increasing the time between settlement pulses (i.e., $\Delta$ ) increased spatial heterogeneity; however, when $v$ was large, increasing the time between pulses decreased heterogeneity (Fig. 4). For the calculations of these critical values and limits, see Appendix E.

\section{Application to a realistic system}

We sought to define plausible parameter regimes to guide our theoretical analyses above. We also sought to evaluate the potential importance of settlement redirection in the context of a real system. To do this, we turned to reef fish as a model system (Table 1). We used data collected from field studies in Moorea, French Polynesia (Shima and Osenberg 2003; Stier and Osenberg 2010), as well as from a meta-analysis of density-dependent mortality in reef fish (Osenberg et al. 2002b).

To estimate parameters that governed redirection (Eq. 3: larval density, $L$; larval survival rate, $\mu$; coral signal decay rate, $\rho$; and the detection threshold, $\theta$ ), we used settlement

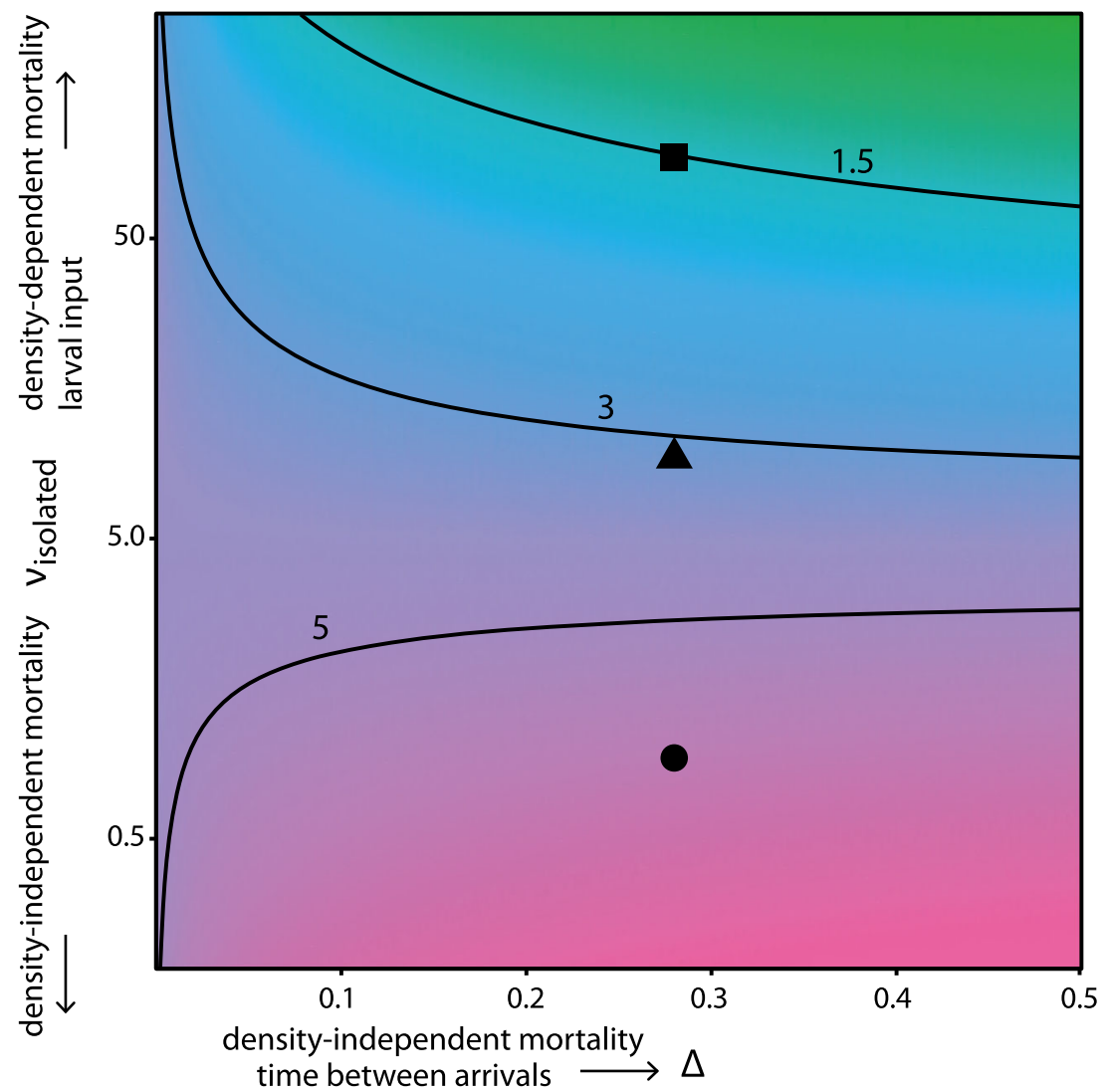

Fig. 4 Relative density of occupants on an isolated coral versus one surrounded by other corals (clustered), $N_{\text {isolated }}^{*} / N_{\text {clustered }}^{*}$, as indicated by the color of each pixel and expressed as a function of the two non-dimensionalized parameters, $v$ and $\Delta$. Initial settlement densities were approximately 17.9:1 (isolated:clustered): The color of each pixel represents the magnitude of the ratio between the equilibrium of a completely isolated and a completely clustered coral. Green pixels (values closer to 1) indicate that there is little spatial variation in occupant density (despite the 17.9-fold variation in settlement). This homogeneous pattern is primarily facilitated by increased density dependence (increasing $v$ ) and increased larval supply (increasing $L)$. Pink pixels (indicating $N_{\text {isolated }}^{*} \gg N_{\text {clustered }}^{*}$ ) indicates that the spatial variation in settlement persists over the long term. The three points give the results from Fig. 3 (circle: $v_{\text {isolated }}=0.9$, triangle: $v_{\text {isolated }}=9$, square: $\left.v_{\text {isolated }}=90\right)$. Contour lines indicate $N_{\text {isolated }}^{*} / N_{\text {clustered }}^{*}=1.5,3$, and 5 
data of four fish species to isolated pairs of corals, pairs of central corals surrounded by a circle of ten neighboring corals, and the ten neighboring corals (Stier and Osenberg 2010). We expressed larval density, $L$, as a function of the number of settlers on the isolated pair and then used the data from other corals to estimate coral signal parameters ( $\rho$ and $\theta$ ) and the larval survival rate $(\mu)$. We searched parameter space using Latin hyper-cube samples and minimized the difference between the observed and simulated values. However, there are more parameters (i.e., four) than reference points (i.e., three types of corals: isolated, central, and circle), so we present the results from one possible parameter set, although there was little difference in final results when we evaluated other parameter options.

The estimation of post-settlement processes are described by parameters: $\alpha$, the density-independent mortality rate, and $\beta$, the density-dependent mortality rate. We obtained an estimate of density-independent mortality, $\alpha=$ 0.0001 , from a meta-analysis for reef fish (Osenberg et al. 2002b). That meta-analysis also provided an estimate of density-dependent mortality: $\beta=0.0005$. In addition, we also used an estimate of density-dependent mortality from a detailed study conducted in Moorea on one species of reef fish, Thalassoma hardwicke (Shima and Osenberg 2003), $\beta=0.056$. We consider this latter estimate to represent very strong density dependence (and thus a worst-case scenario for settlement redirection persisting through time), whereas the estimate from the meta-analysis is a more typical (moderate) estimate.

The application also required that we specify a spatial landscape. While previously we fixed the number of corals in our simulations to focus solely on effects due to configuration, landscapes with more corals have higher potential for interactions among patches than landscapes with few patches (that are likely more isolated). Therefore, we wanted a landscape that was realistic in both the density of patches and patch configuration. Because we were using studies from Moorea to motivate our analyses, we used Google Earth to identify coral habitat on a $1000-\mathrm{m}^{2}$ area of backreef of the north shore of Moorea. We then overlaid this habitat area with circles of $1-\mathrm{m}^{2}$ radii to define units of habitat within which fish potentially settled and interacted.

In addition to propagule redirection, prior work in the Moorea system suggests that spatial variation in habitat quality can produce spatial variation in density-dependent mortality and settlement: high quality sites receive more settlers and incur lower levels of density-dependent mortality (Shima and Osenberg 2003). This covariance between density dependence and settlement generates a pattern called "cryptic density dependence" (CDD: see Wilson and Osenberg (2002) and Shima and Osenberg (2003)). For our purposes, we wanted to include possible effects of settlement redirection as well as other mechanisms that could produce spatial variation or that might mask or alleviate the effects of propagule redirection. Therefore we simulated dynamics under four scenarios: (i) a control (i.e., no propagule redirection or variation in density-dependent mortality), which served as a baseline for comparison; (ii) the presence of spatial variation in habitat quality, but not propagule redirection (i.e., covariance between settlement cues and density dependence as articulated in CDD); (iii) the presence of propagule redirection only (i.e., variable settlement due to redirection, but spatially uniform habitat quality); and (iv) the presence of both propagule redirection and variation in habitat quality (i.e., spatially variable settlement due to redirection and settlement cues, and variable density dependence due to CDD).

To simulate spatial variation in density dependence, we sampled from the distribution of $\beta$ from Shima and Osenberg (2003) which had a mean of 0.056 (for strong density dependence) or from a distribution with those values divided by 1000 (thus achieving a mean of 0.00056 : i.e., moderate density dependence that was comparable to the mean from the meta-analysis of Osenberg et al. (2002a)). Thus, with only propagule redirection, density dependence was spatially uniform (with $\beta=0.056$ or $\beta=0.00056$ ), whereas with habitat quality, the mean level of density dependence was the same, albeit spatially variable. To represent spatial variation in settlement due to habitat quality, we assumed that expected settlement to a coral was proportional to $1 / \beta$ (as is the case in CDD: Shima and Osenberg (2003)) and then adjusted the signal to yield the same average signal as in the propagule redirection scenario.

We therefore evaluated eight scenarios ([presence vs. absence of spatial variation in habitat quality] $X$ [presence vs. absence of propagule redirection] $\mathrm{X}$ [strong $(\beta=$ $0.056)$ vs. moderate $(\beta=0.00056)$ mean density dependence]). For each scenario, we simulated settlement and post-settlement dynamics, as in the previously described stochastic simulations, recorded the index of dispersion for settlement and occupants, and repeated this 100 times.

Both propagule redirection and CDD generated spatial variation in settlement compared to the null scenario when applied to the realistic Moorea landscape (Fig. 5a). Heterogeneity due to propagule redirection was 1.6 times greater than generated by CDD (i.e., habitat quality) alone. Additionally, when we included effects of both habitat quality (CDD) and settlement redirection, the resulting settlement pattern had an index of dispersion 2.1 times greater than that produced by CDD alone and 1.3 times greater than that produced by settlement redirection alone. Thus, settlement redirection played a comparable or larger role in determining settlement patterns than spatial variation in habitat quality.

Density dependence is generally assumed to reduce spatial variation caused by settlement, and this was the case 

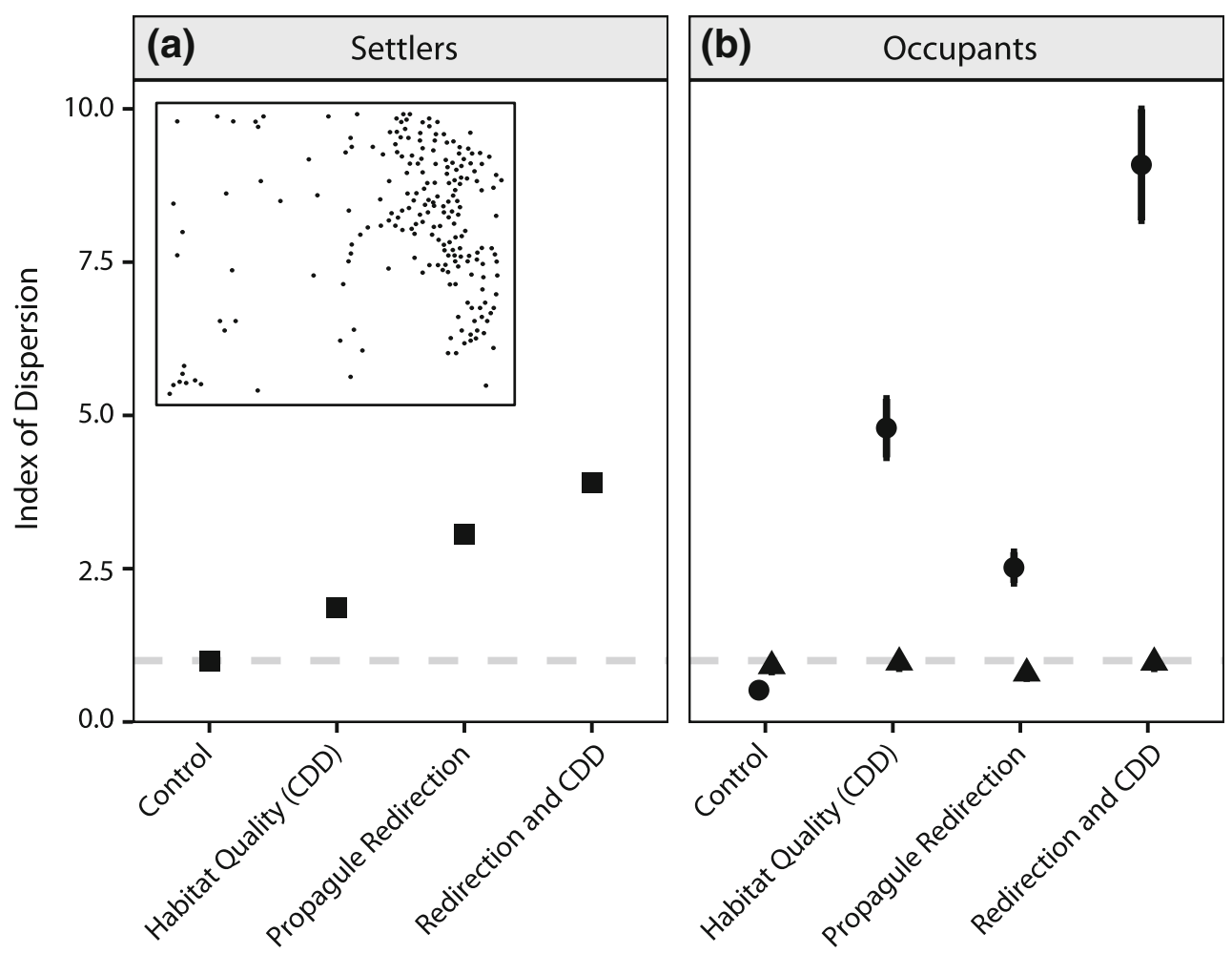

Treatment

Fig. 5 Spatial heterogeneity in a settlers and $\mathbf{b}$ occupants for a portion of the backreef of the north shore of Moorea, French Polynesia (inset of a). An index of dispersion (variance/mean) $>1$ indicates a distribution more heterogeneous than random (i.e., Poisson) for the focal life stage, $=1$ indicates a random spatial distribution (dashed horizontal line), and $<1$ indicates a more even distribution. Propagule redirection creates slightly more heterogeneity in settlement than variation in habitat quality alone (as captured by the phenomenon of

when density dependence was strong (Fig. 5b). In all scenarios, the index of dispersion was close to 1 , indicating that occupant density was spatially variable, but not distinguishable from that expected under a Poisson spatial processes. This was true even when density dependence was spatially variable (as in CDD). In contrast, under moderate densitydependent mortality, there was greater variation in occupant density than expected by a Poisson process, except in the control scenario. For example, in the presence of propagule redirection (and spatially uniform density dependence), the spatial variation in settlement was maintained for the occupants. In the presence of spatial variation in densitydependence, the index of dispersion actually increased, intensifying spatial variation in the density of occupants, and the combination of redirection and CDD was 1.9 times that of CDD alone or 3.6 times that of redirection alone.

These simulations demonstrated that landscape configuration (which creates variation in the proximity of neighboring patches) and spatial variation in settlement cues (due cryptic density dependence; Shima and Osenberg (2003)). Under moderate ( $\beta=0.00056$ ) density-dependent mortality (circles), propagule redirection increased the heterogeneity in occupants by 1.6 -fold compared to differences due quality alone. Under strong $(\beta=0.056)$ density-dependent mortality (triangles), variation among patches was not distinguishable from a Poisson spatial process. In all simulations, we set $\mu=0.4, \rho=0.75, \alpha=0.0001 \gamma=28, R=1$, and $L=0.33$. Error bars represent $95 \%$ CIs

to differences in habitat quality) create approximately equal spatial variation in settlement. Under moderate (and weak) density dependence, these settlement patterns create longterm patterns of occupant density that are equally variable (when density dependence is spatially uniform) or magnified in intensity (when density dependence is spatially variable).

\section{Discussion}

Heterogeneity in the number of occupants among habitat patches is often attributed to intrinsic characteristics of the patches. However, here we demonstrate that the configuration of patches in a landscape also can create spatial heterogeneity in colonization and resident density among otherwise identical patches. Therefore, the spatial distribution of patches alone can cause long-term heterogeneity in a system independent of other factors. 
This heterogeneity requires that habitat patches vary in their proximity to other patches and that this proximity reduces the input of colonists to these patches. In such a system, landscapes with a few isolated patches and many clustered patches will exhibit far greater heterogeneity than a landscape where all patches are similarly spaced. The phenomenon in which patch configuration creates settlement heterogeneity, propagule redirection, has not been investigated widely. Stier and Osenberg (2010) found that neighboring corals reduced the settlement of reef fishes to focal corals. Morton and Shima (2013) found a similar pattern in a temperate fish when comparing discrete habitat patches (i.e., but not when comparing single patches of increasing size). Other marine organisms display related spatial patterns of settlement. For example, larvae of the intertidal barnacle, Balanus glandula, show spatial heterogeneity in settlement due to depletion of larvae from the water column. Downstream sites had lower settlement, either because larval densities were depleted as larvae settled upstream (Gaines et al. 1985) or because predators reduced larval density (Gaines and Roughgarden 1987). Upstream sites have also been shown to "steal" larvae from downstream sites in a coral reef system on the Great Barrier Reef: fish recruitment was diminished on corals immediately downstream of other suitable corals (Jones 1997). These settlement shadows (sensu Jones (1997)) are analogous to patterns created via propagule redirection. We note, however, that these examples of settlement shadows largely presume directional supply of larval leading to a depletion of larval stocks from upstream to downstream areas. This phenomenon would act in consort with propagule redirection to generate spatial variation in settlement: in the extreme, settlement shadows deplete larval density ( $L$ in our model) creating an upstream-downstream gradient in settlement, while propagule redirection would promote variation in settlement perpendicular to flow (i.e., creating variation in settlement for a given larval density).

The above evidence comes from marine organisms in which larvae are the dispersive stage. Propagule redirection is likely in other systems as well. For example, many freshwater organisms have a dispersive adult stage but a relatively sedentary larval stage: e.g., aquatic insects such as mosquitoes or odonates have dispersing adults that oviposit in or colonize ponds and lakes. In these systems, the availability and distribution of ponds could generate heterogeneity: ponds with many neighboring ponds may attract fewer ovipositing females or colonizing adults. However, experiments with aquatic beetles showed that colonization rates were independent of local pond (patch) density among patches without predators (Resetarits and Binckley 2009; 2013). It is not clear what produced these disparate results, but it is likely that the degree of propagule redirection will depend on the scale of movement of the dispersive stage, the scale of their sensory abilities (how far they can discern among habitat patches), the spacing of habitats in the landscape (relative to movement and sensory abilities), and the mortality incurred moving across the landscape. It is possible that beetles and reef fish experiments were conducted at different relative levels of these factors. Further research should investigate how the relative scales of these processes produce differences in the spatial patterns of colonization.

Spatial scale can also alter expected patterns because organisms make choices at multiple spatial scales. In our analyses, we have focused on a relatively small (or local) scale, in which the density of potential colonists was spatially uniform (and reflected in larval density, $L$ ). However, at a larger scale (a "region"), colonists may be attracted to regions with more patches. Thus, regions with many patches could draw in more potential colonists, but within a region, propagule redirection could generate variation in colonization: i.e., at one scale (the region), neighbors draw in more colonists, but at a smaller scale (patch), neighbors reduce colonization. This is analogous to what happens with pollinators: more apparent floral displays (or higher plant densities) recruit more pollinators, but within a flowering patch, a lower proportion of flowers are visited as flowers compete for pollinators (e.g., Ohashi and Yahara (1999) and Grindeland et al. (2005)). Resolving the relative importance of these processes (and the sensory cues used at these different scales) will be an important next step.

Heterogeneous patterns established by redirection and the arrangement of patches within a region are most likely to persist when the system is undersaturated, due either to low density-dependent mortality, high density-independent mortality, a limited supply of colonists, or a short life-span (which reduces the number of co-occurring cohorts within a patch). As a system nears saturation, the distribution of occupants in the system becomes more homogeneous, obliterating the spatial patterns first established during the colonization phase. The linkage between patterns established at settlement and those that persist at later life stages has a long history in "supply-side ecology" (e.g., Gaines et al. (1985)). While these studies are typically at a much larger spatial scale, another difference between that tradition and what we studied here is that the settlement patterns in supply-side ecology are often attributed to stochastic processes (e.g., due to unusually large settlement events) or intrinsic properties of sites (e.g., that lead to greater settlement). Here, the neighborhood itself generates spatial variation in settlement.

The effects that neighboring patches have on settlement, and resulting in long-term spatial patterns, have important implications for applications such as habitat restoration. Many restoration techniques focus not on the target organisms but instead on the restoration of their habitat. For 
example, flower patches are added for pollinators (e.g., Wratten et al. (2012)), and reef structures are created for marine invertebrates and fish (e.g., Burt et al. (2009)). If habitat is added to an area, the expectation is that the added habitat will attract colonists and help re-establish the target population. However, if redirection occurs, this additional habitat might not lead to a higher density of organisms - it may simply attract colonists away from the existing habitat (Pickering and Whitmarsh 1997), and if density dependence is weak, there may be little net benefit of the habitat restoration on the focal species (Carr and Hixon 1997; Osenberg et al. 2002a).

Although we incorporated realism into our application by applying our approach to a realistic landscape, our overall approach ignored several complexities of real systems that allowed us to focus on the effect of neighbors. Not only did we focus on local among-patch competition for colonists (see above discussion about spatial scale) but we also made simplifying assumptions with regard to patch characteristics. For example, we assumed that all patches were uniform in size, although patch size is an important variable that can affect colonization. Patch size and isolation of a patch affects colonization of ponds by amphibians (Laan and Verboom 1990) and aquatic insects (Wilcox 2001). As illustrated in our application to Moorea, habitat quality (and associated signal) also likely varies and leads to differences in colonization (e.g., Holbrook et al. 2000; Resetarits and Binckley 2009, 2013; Resetarits and Silberbush 2016; Shima and Osenberg, 2003). Furthermore, signals can vary within and between types of habitats (Dixson et al. 2014). Empirically, we might expect a correlation between the strength of these signals and habitat quality. This may be especially important for biogenic habitat (such as corals or trees), in which the habitat patches either mask their signals (to hide from colonists that harm that habitat) or enhance their signals (to attract beneficial colonists). Our realistic landscape simulations indicated that variation in signal strength will likely intensify spatial heterogeneity in settlement (Fig. 5).

Finally, we assumed that the intrinsic qualities of the patches (e.g., size) were static throughout time. While this may be true for some habitats, many biogenic habitat patches (e.g., corals, trees) are dynamic, and as a result, their characteristics change through time. Their dynamics can be affected by many processes (including competition, predation and disease dynamics) that drive spatial pattern in the growth and survival of the organisms that occupy the biogenic habitat. For example, coral symbionts (e.g., fish or crabs) increase coral growth and survival by providing nutrients to the coral (Holbrook et al. 2008, 2011) and by defending the coral from predators or other harmful factors (Glynn 1980; Pratchett et al. 2000; Stier et al. 2010). As a result, we might expect clusters of patches to do poorly, not because they compete for food and not because of increased disease transmission but instead because they compete for beneficial symbionts. This form of competition, driven by propagule redirection, may be an important process in systems in which biogenic habitat harbors beneficial symbionts that have open demographics.

Acknowledgments We thank the Osenberg lab and Shilpa Khatri for helpful discussions and the National Science Foundation (OCE-1130359, OCE-0242312) and the QSE3 IGERT program (DGE0801544) and Army Research Office (Grant Number 64430-MA) for support. We would also like to thank Ben Bolker, Alan Hastings, and anonymous reviewers for feedback on an earlier version of this manuscript.

\section{Appendix}

\section{A. Comparison of the ODE approximation to the mean of the stochastic model}

As described in the "Methods" section, we use a stochastic and a deterministic model in tandem to study settlement shadow dynamics. Because these mathematical systems are non-linear (by virtue of the density-dependent mortality), the deterministic model is not simply the mean of the stochastic version. In fact, the deterministic model systematically underestimates the mean of the stochastic process due to an application of Jensen's inequality. We use this subsection to justify this claim (Fig. 6).

The stochastic model is a continuous-time Markov chain denoted $N(t)$. For a given initial condition $n \in\{0,1,2 \ldots\}$, we define a collection of functions $\left\{p_{k}(t)\right\}_{k \geq 0}$ that respectively describe the probability that there are $k$ settlers on a focal coral at time $t$,

$$
p_{k}(t)=\mathbb{P}_{n}(N(t)=k)=\mathbb{I} N(t)=k \mid N(0)=n .
$$

Recalling that $\gamma$ is the time between settlement pulses, we can write the master equations for this Markov chain for all $t \in[0, \gamma)$ as follows:

$$
\begin{aligned}
\frac{d}{d t} p_{k}(t)= & -\left(\alpha k+\beta k^{2}\right) p_{k}(t)+\left(\alpha(k+1)+\beta(k+1)^{2}\right) \\
& \times p_{k+1}(t)+\delta_{0}(t) \sum_{n=0}^{k} \mathbb{\llbracket} \lambda=k-n p_{n}(0) .
\end{aligned}
$$

The first term on the right-hand side results from the rate of the transition $k \rightarrow k-1$ while the second term corresponds to the transition $k+1 \rightarrow k$. Noting that the size of the initial pulse $\lambda$ is a Poisson distributed random variable, the last term captures the effect of the initial pulse $\left(\delta_{0}(t)\right.$ is a Dirac-delta function concentrated at the initial time $t=0$ ). 
Fig. 6 A comparison of the stochastic model (described in "Post-settlement processes") and the analytic approximation for post-settlement mortality (4) a Small population sizes started with $\lambda=50$ and $\mathbf{b}$ large populations with $\lambda=500$. At large population sizes, the analytic approximation nicely approximates the stochastic process. However, at small populations sizes, the approximation underestimates density-dependent mortality (a)

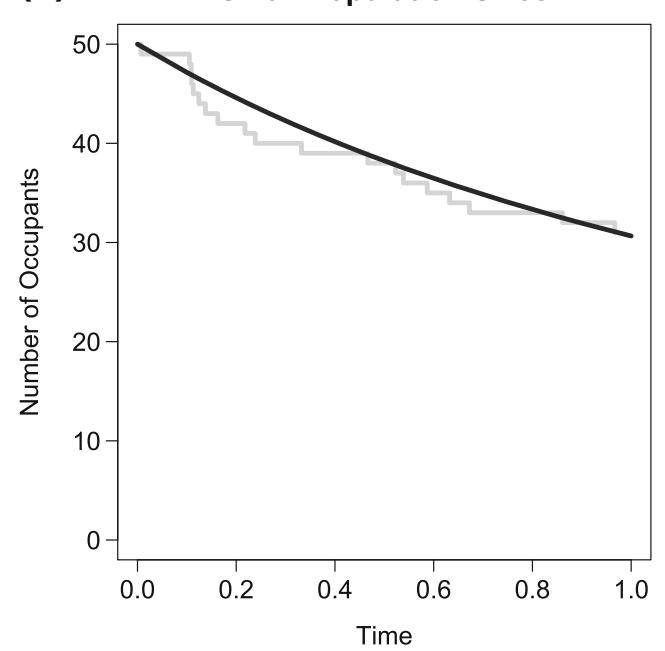

(b) Large Population Sizes

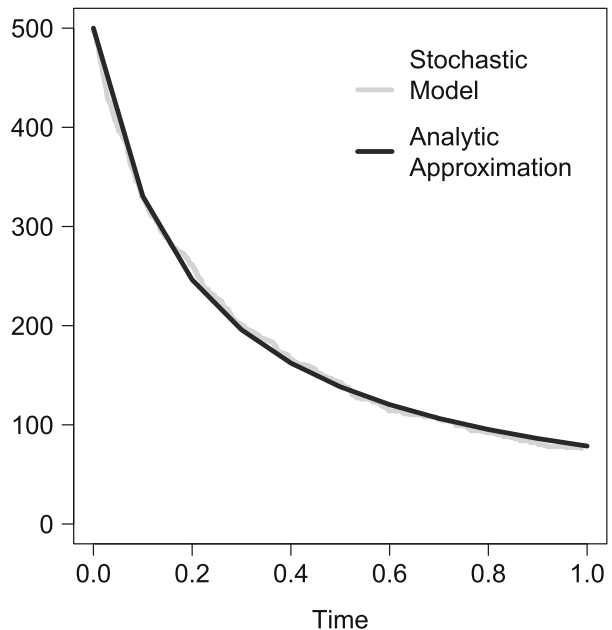

After the initial pulse has occurred, we can look at the derivative of the mean of the stochastic model conditioned on having the initial value $n$, (denoted $\mathbb{E}_{n}[N(t)]$ ),

$$
\begin{aligned}
\frac{d}{d t} \mathbb{E}_{n}[N(t)]= & \frac{d}{d t} \sum_{k=1}^{\infty} k p_{k}(t) \\
= & \sum_{k=1}^{\infty} k\left[-\left(\alpha k+\beta k^{2}\right) p_{k}(t)+(\alpha(k+1)\right. \\
& \left.\left.\quad+\beta(k+1)^{2}\right) p_{k+1}(t)\right] \\
= & -\sum_{k=1}^{\infty}\left(-\alpha k-\beta k^{2}\right) p_{k}(t) \\
= & -\alpha \mathbb{E}_{n}[N(t)]-\beta \mathbb{E}_{n}\left[N^{2}(t)\right]
\end{aligned}
$$

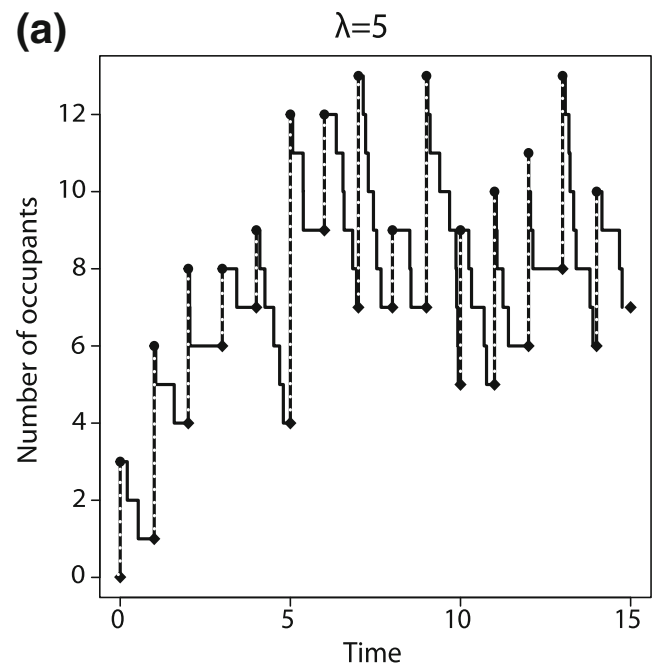

Fig. 7 The stochastic dynamics of fish within a single coral under a low and $\mathbf{b}$ high settlement intensities. New fish arrive during discrete settlement events (circles) that are followed by continuous (but stochastic) declines in abundance (solid lines) until just before the arrival of another settlement event (diamonds). Empty corals
By Jensen's Inequality, we observe that

$-\alpha \mathbb{E}_{n}[N(t)]-\beta \mathbb{E}_{n}\left[N^{2}(t)\right] \leq-\alpha \mathbb{E}_{n}[N(t)]-\beta\left(\mathbb{E}_{n}[N(t)]\right)^{2}$

Now, note that the right-hand side of this inequality is precisely the form seen in the ODE we use in our deterministic approximation $\bar{N}(t)$ defined by Eq. 4 :

$\bar{N}(t)^{\prime}=-\alpha \bar{N}(t)-\beta(\bar{N}(t))^{2}$.

This implies that the mean of the stochastic process $N(t)$ is decreasing faster than the approximating deterministic process $\bar{N}(t)$. For larger populations, this effect is negligible, but this may not be the case in the dynamics of smaller populations.

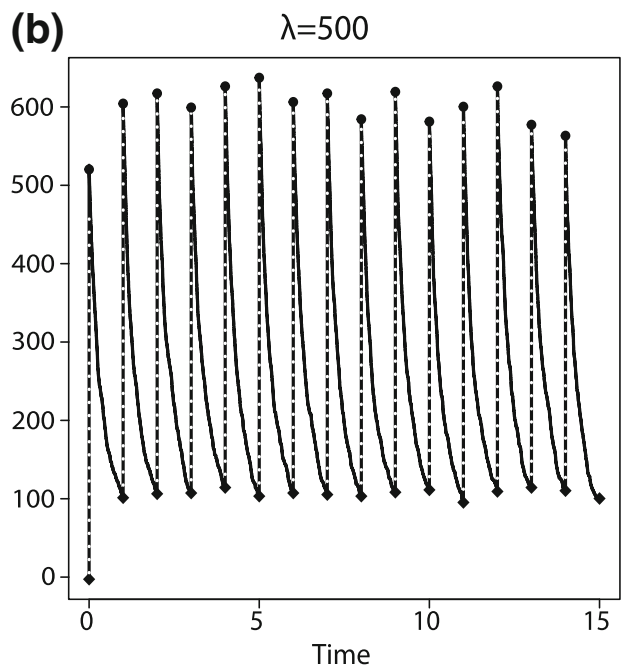

accumulate larvae and eventually converge on an equilibrium at which the number of settlers equals the number of deaths (of recent settlers and older fish) during the inter-pulse period. At high larval densities, the stochastic dynamics resemble a continuous approximation. In both panels, $\alpha=0.5, \beta=0.005$, and $\gamma=1$ 


\section{B. Convergence of the ODE to equilibrium}

As seen in right panel of Fig. 7, when the population is relatively large, there is a stable recurring pattern in the time periods marked initially by a settlement pulse and followed by a mortality phase. To assign a single number for this pattern, we focus on the sequence of values $\{N(i \Delta)\}_{i=0}^{\infty}$ (Eq. 8) corresponding to the population sizes just before settlement pulses occur. Over time, in the deterministic model, these values converge to a long-term steady value, $\tilde{N}^{*}$. To compute this quantity, we found the solution to the ODE (Eq. 6) between settlement pulses and then note that periodic equilibrium occurs with the number of deaths that occur in the time between settlement pulses is equal to the size of the pulses. That is to say, we look for the point when the sequence of values $\{N(i \Delta)\}_{i=0}^{\infty}$ (Eq. 8) satisfies the condition $N((i+1) \Delta)=\varphi(N(i \Delta))$ where

$\varphi(x)=\frac{(x+v) e^{-\Delta}}{1+(x+v) e^{-\Delta}}$.

The function has one fixed point and the formula is given by Eq. 8. Furthermore, because $\varphi$ is increasing and concave down, for all $x<\tilde{N}^{*}$ we have $\varphi(x)>x$. On the other hand, if $x>\tilde{N}^{*}$, then $\varphi(x)<x$. It follows that $\{N(i \Delta)\}_{i=0}^{\infty}$ is a bounded, monotone sequence of points and therefore the sole fixed point is asymptotically stable.

\section{Expected number of settlers on an isolated and a clustered coral}

In our system, colonists arrive as a spatial Poisson process, which is to say that the total number drawn has a Poisson distribution and the location of each is drawn uniformly at random from the domain. The landscape contains circular patches with radius $R$, the centers of which are generated by one of the three spatial point processes (even, random, clustered) described in the main text. Within these landscape configurations, the patches have varying degrees of isolation. The most extreme cases occur when a patch is completely isolated or completely surrounded by other patches. We can analytically compute the exact formula for the distribution of the larval input in these two extreme cases.

In the case of the completely clustered patch, the expected larval input, $\lambda_{\text {clustered }}$ includes only settlers that arrive directly on the coral. Since colonists arrive uniformly at random with density $L$, the mean number of arrivals is simply the density times the area of the patch:

$\lambda_{\text {clustered }}=L \pi R^{2}$.

Calculating the expected colonist input to a completely isolated coral is more complicated. Suppose there is a circular coral patch of radius $R$ centered at the origin of the $x-y$ plane. We compute the expected number of settlers that arrive at the coral assuming that their initial location in the water column is uniformly distributed over a circular landscape, $\mathcal{O}$, centered at the origin and having radius $R_{\mathcal{O}}$. However, due to the detection threshold, $\theta$, we only consider the area to $R_{*}$, or the radius where the signal is detectable, or where $\theta=m e^{-\rho\left(R_{*}-R\right)}$. Thus, we integrate to $R_{*}=\frac{\ln (m / \theta)}{\rho}+R$.

In this setting, Eq. 3, which describes the probability that any given settler arrives at the focal coral, takes the form

$$
\begin{aligned}
p\left(R_{*}\right) & :=\frac{1}{|\mathcal{O}|} \iint_{\mathcal{O}} \frac{\Lambda_{1}(x, y)}{\sum_{j} \Lambda_{j}(x, y)} e^{-\mu d_{1}(x, y)} d x d y \\
& =\frac{R^{2}}{R_{*}^{2}}+\frac{1}{\pi R_{\mathcal{O}}^{2}} \int_{\theta=0}^{2 \pi} \int_{r=R}^{R_{*}} e^{-\mu(r-R)} r d r d \theta
\end{aligned}
$$

where the first term is the probability that a settler descends into the water column directly onto the coral and the second term includes the possibility of mortality in the travel from the initial location to the coral. Integrating, we find

$p\left(R_{*}\right)=\frac{R^{2}}{R_{*}^{2}}+\frac{\mu R+1-\left(\mu R_{*}+1\right) e^{\mu\left(R-R_{*}\right)}}{\mu^{2}}$

Substituting $R_{*}=\frac{\ln (m / \theta)}{\rho}+R$,

$$
\begin{aligned}
p\left(R_{*}\right)= & \frac{\rho^{2} R^{2}}{\ln (m / \theta)^{2}}+\frac{2 \pi}{\mu^{2}} \\
& \times\left[\mu R+1-\left(\frac{m}{\theta}\right)^{-\mu / \rho}\left(\frac{\mu}{\rho} \ln \left(\frac{m}{\theta}\right)+\mu R+1\right)\right]
\end{aligned}
$$

The number of settlers that arrive at the focal coral is then a thinned Poisson process with mean $L\left|\pi R_{*}^{2}\right| p\left(R_{*}\right)$

$$
\begin{aligned}
\lambda_{\text {isolated }}= & L \pi R^{2}+\frac{2 \pi L}{\mu^{2}} \\
& \times\left[\mu R+1-\left(\frac{m}{\theta}\right)^{-\mu / \rho}\left(\frac{\mu}{\rho} \ln \left(\frac{m}{\theta}\right)+\mu R+1\right)\right] .
\end{aligned}
$$

\section{Non-dimensionalization}

To non-dimensionalize the ODE (4), we introduce the following rescaled quantities:

$\tau=\alpha t, d \tau=\alpha d t, \tilde{N}(\tau)=\bar{N}(t) /(\alpha / \beta)$, and $\tilde{\delta}(\tau)=\delta(t) / \alpha$.

Differentiating $\tilde{N}$ with respect to $\tau$ is related to $d \bar{N} / d t$ by the relations

$\frac{d}{d \tau} \tilde{N}(\tau)=\frac{d}{d \tau} \frac{\bar{N}}{\alpha / \beta}=\frac{1}{\alpha} \frac{d}{d t} \frac{\bar{N}}{\alpha / \beta}=\frac{1}{\alpha^{2} / \beta} \frac{d \bar{N}}{d t}$. 
It follows that

$$
\begin{aligned}
\frac{d \tilde{N}}{d \tau} & =\frac{1}{\alpha^{2} / \beta}\left[\alpha \bar{N}(t)-\beta \bar{N}^{2}(t)+\sum_{k=0}^{\infty} \bar{\lambda} \delta_{k \gamma k}(t)\right] \\
& =\frac{1}{\alpha^{2} / \beta}\left[\left(-\frac{\frac{\alpha}{\beta} \alpha \bar{N}(t)}{\alpha / \beta}\right)-\frac{\frac{\alpha^{2}}{\beta^{2}} \beta \bar{N}^{2}(t)}{\frac{\alpha^{2}}{\beta^{2}}}+\sum_{k=0}^{\infty} \bar{\lambda} \delta_{k \gamma k}(t)\right] .
\end{aligned}
$$

If we substitute $\tilde{N}(\tau)=\bar{N} / \alpha / \beta$, then we have

$$
\frac{d \tilde{N}}{d \tau}=-\tilde{N}(\tau)-\tilde{N}^{2}(\tau)+\frac{\bar{\lambda}}{\alpha^{2} / \beta} \sum_{k=0}^{\infty} \delta(t-\gamma k) .
$$

Next, we introduce the non-dimensionlized $\tilde{\delta}(\tau)=\delta(t) / \alpha$

$$
\begin{aligned}
\frac{d \tilde{N}}{d \tau} & =-\tilde{N}(\tau)-\tilde{N}^{2}(\tau)+\frac{\bar{\lambda}}{\alpha^{2} / \beta} \sum_{k=0}^{\infty} \alpha \tilde{\delta}(\alpha(t-\gamma k)) \\
& =-\tilde{N}(\tau)-\tilde{N}^{2}(\tau)+\frac{\bar{\lambda}}{\alpha / \beta} \sum_{k=0}^{\infty} \tilde{\delta}(\alpha t-\alpha \gamma k) .
\end{aligned}
$$

Finally, introducing $v=\beta \bar{\lambda} / \alpha$ and $\Delta=\alpha \gamma$, we express the ODE in completely non-dimensional terms:

$$
\frac{d \tilde{N}}{d \tau}=-\tilde{N}(\tau)-\tilde{N}^{2}(\tau)+v \sum_{k=0}^{\infty} \tilde{\delta}_{\Delta k}(\tau) .
$$

\section{E. Effect of $\Delta$ on spatial heterogeneity}

The effect of time between settlement pulses relative to the settlement rate depended on $v$. There was a critical value, $v_{\text {isolated }}=\sqrt{\frac{\lambda_{\text {isolated }}}{\lambda_{\text {clustered }}}}$, at which changing $\Delta$ had no effect on the resulting heterogeneity. Instead, at this critical value of $v$, the variation in occupant numbers (between an isolated and clustered coral) is constant and equal to $\sqrt{\frac{\lambda_{\text {isolated }}}{\lambda_{\text {clustered }}}}$ (i.e., heterogeneity was present, but reduced from that imposed at settlement). In addition, as $\Delta \rightarrow 0$ the relative density of occupants approached this same value (i.e., $\sqrt{\frac{\lambda_{\text {isolated }}}{\lambda_{\text {clustered }}}}$, and as $\Delta \rightarrow \infty$, the relative density approached a constant, $\frac{v_{\text {isolated }}\left(1+v_{\text {clustered }}\right)}{v_{\text {clustered }}\left(1+v_{\text {isolated }}\right)}$. Thus, the effects of increased density-independent mortality and time between arrivals (which affect $\Delta$ ) cannot be viewed through the same lens as we viewed the effects of $v$. Although increasing $\Delta$ suggests a reduction in saturation, the homogenizing effects of density dependence still operate immediately following settlement pulses. Thus, increasing $\Delta$ cannot strictly maintain the spatial variation created by propagule redirection; some homogenization will be achieved (except in the limiting case as $v \rightarrow 0$ ).

\section{References}

Baddeley A, Turner R (2005) Spatstat: an R package for analyzing spatial point patterns. J Stat Softw 12(6):1-42. www.jstatsoft.org, ISSN: 1548-7660

Burt J, Bartholomew A, Usseglio P, Bauman A, Sale P (2009) Are artificial reefs surrogates of natural habitats for corals and fish in Dubai, United Arab Emirates Coral Reefs 28(3):663-675

Carr MH, Hixon MA (1997) Artificial reefs: the importance of comparisons with natural reefs. Fisheries 22(4):28-33

Dixson DL, Abrego D, Hay ME (2014) Chemically mediated behavior of recruiting corals and fishes: a tipping point that may limit reef recovery. Science 345(6199):892-897

Eaton MJ, Hughes PT, Hines JE, Nichols JD (2014) Testing metapopulation concepts: effects of patch characteristics and neighborhood occupancy on the dynamics of an endangered lagomorph. Oikos 123(6):662-676

Gaines S, Brown S, Roughgarden J (1985) Spatial variation in larval concentrations as a cause of spatial variation in settlement for the barnacle, Balanus glandula. Oecologia 67(2):267-272

Gaines SD, Roughgarden J (1987) Fish in offshore kelp forests affect recruitment to intertidal barnacle populations. Science 235(4787): $479-480$

Gillespie DT (1977) Exact stochastic simulation of coupled chemical reactions. J Phys Chem 81(25):2340-2361

Glynn PW (1980) Defense by symbiotic crustacea of host corals elicited by chemical cues from predator. Oecologia 47(3):287-290

Gonzalez A, Lawton J, Gilbert F, Blackburn T, Evans-Freke I (1998) Metapopulation dynamics, abundance, and distribution in a microecosystem. Science 281(5385):2045-2047

Grindeland JM, Sletvold N, Ims RA (2005) Effects of floral display size and plant density on pollinator visitation rate in a natural population of digitalis purpurea. Funct Ecol 19(3):383-390

Gustafson EJ, Gardner RH (1996) The effect of landscape heterogeneity on the probability of patch colonization. Ecology 77(1):94107

Hanski I (1994) A practical model of metapopulation dynamics . J Anim Ecol 63:151-162

Hein AM, McKinley SA (2013) Sensory information and encounter rates of interacting species. PLoS Comput Biol 9(8):e1003,178. https://doi.org/10.1371/journal.pcbi.1003178

Hill J, Thomas C, Lewis O (1996) Effects of habitat patch size and isolation on dispersal by Hesperia comma butterflies: implications for metapopulation structure. J Anim Ecol 65:725-735

Hixon MA, Jones GP (2005) Competition, predation, and densitydependent mortality in demersal marine fishes. Ecology 86(11): 2847-2859

Holbrook S, Schmitt R (1997) Settlement patterns and process in a coral reef damselfish: in situ nocturnal observations using infrared video. In: Proceedings of the 8th International Coral Reef Symposium, vol 2, pp 1143-1148

Holbrook S, Forrester G, Schmitt R (2000) Spatial patterns in abundance of a damselfish reflect availability of suitable habitat. Oecologia 122(1):109-120

Holbrook S, Brooks A, Schmitt R, Stewart H (2008) Effects of sheltering fish on growth of their host corals. Mar Biol 155(5):521530

Holbrook S, Schmitt R, Brooks A (2011) Indirect effects of species interactions on habitat provisioning. Oecologia 166(3):739-749

Holbrook SJ, Schmitt RJ (2002) Competition for shelter space causes density-dependent predation mortality in damselfishes. Ecology 83(10):2855-2868

Jones G (1997) Relationships between recruitment and postrecruitment processes in lagoonal populations of two coral reef fishes. J Exp Mar Biol Ecol 213(2):231-246 
Jones G, Almany G, Russ G, Sale P, Steneck R, Van Oppen M, Willis B (2009) Larval retention and connectivity among populations of corals and reef fishes: history, advances and challenges. Coral Reefs 28(2):307-325

Koelle K, Vandermeer J (2005) Dispersal-induced desynchronization: from metapopulations to metacommunities. Ecol Lett 8(2):167-175

Laan R, Verboom B (1990) Effects of pool size and isolation on amphibian communities. Biol Conserv 54(3):251-262

Lecchini D, Adjeroud M, Pratchett MS, Cadoret L, Galzin R (2003) Spatial structure of coral reef fish communities in the Ryukyu Islands, southern Japan. Oceanol Acta 26(5):537-547

Lecchini D, Shima J, Banaigs B, Galzin R (2005) Larval sensory abilities and mechanisms of habitat selection of a coral reef fish during settlement. Oecologia 143(2):326-334

MacArthur RH, Wilson EO (1967) The theory of island biogeography, vol 1. Princeton University Press, Princeton

Mitchell RJ, Flanagan RJ, Brown BJ, Waser NM, Karron JD (2009) New frontiers in competition for pollination. Ann Bot 103(9): 1403-1413

Mönkkönen M, Helle P, Soppela K (1990) Numerical and behavioural responses of migrant passerines to experimental manipulation of resident tits (Parus spp.): heterospecific attraction in northern breeding bird communites Oecologia 85(2):218-225

Morales CL, Traveset A (2008) Interspecific pollen transfer: magnitude, prevalence and consequences for plant fitness. Crit Rev Plant Sci 27(4):221-238

Morton DN, Shima JS (2013) Habitat configuration and availability influences the settlement of temperate reef fishes Tripterygiidae. J Exp Mar Biol Ecol 449:215-220

Ohashi K, Yahara T (1999) How long to stay on, and how often to visit a flowering plant?: a model for foraging strategy when floral displays vary in size. Oikos 86:386-392

Osenberg C, Mary C, Wilson J, Lindberg W (2002a) A quantitative framework to evaluate the attraction-production controversy. ICES J Mar Sci J du Conseil 59(suppl):S214-S221

Osenberg CW, St Mary CM, Schmitt RJ, Holbrook SJ, Chesson P, Byrne B (2002b) Rethinking ecological inference: density dependence in reef fishes. Ecol Lett 5(6):715-721

Pickering H, Whitmarsh D (1997) Artificial reefs and fisheries exploitation: a review of the 'attraction versus production'debate, the influence of design and its significance for policy. Fish Res 31(1):39-59

Pratchett M, Vytopil E, Parks P (2000) Coral crabs influence the feeding patterns of crown-of-thorns starfish. Coral Reefs 19(1):36-36

Resetarits WJ, Binckley CA (2009) Spatial contagion of predation risk affects colonization dynamics in experimental aquatic landscapes. Ecology 84(4):869-876
Resetarits WJ, Binckley CA (2013) Patch quality and context, but not patch number, drive multi-scale colonization dynamics in experimental aquatic landscapes. Oecologia 173(3):933-946

Resetarits WJ, Silberbush A (2016) Local contagion and regional compression: habitat selection drives spatially explicit, multiscale dynamics of colonisation in experimental metacommunities. Ecol Lett 19(2):191-200

Ricketts TH (2001) The matrix matters: effective isolation in fragmented landscapes. Am Nat 158(1):87-99

Robertson DR (1992) Patterns of lunar settlement and early recruitment in caribbean reef fishes at panama. Mar Biol 114(4):527537

Ryall KL, Fahrig L (2006) Response of predators to loss and fragmentation of prey habitat: a review of theory. Ecology 87(5):10861093

Shima JS, Osenberg CW (2003) Cryptic density dependence: effects of covariation between density and site quality in reef fish. Ecology $84(1): 46-52$

Sih A, Baltus MS (1987) Patch size, pollinator behavior, and pollinator limitation in catnip. Ecology 68:1679-1690

Stamps J (1988) Conspecific attraction and aggregation in territorial species. American Naturalist 131:329-347

Stier A, Osenberg C (2010) Propagule redirection: habitat availability reduces colonization and increases recruitment in reef fishes. Ecology 91(10):2826-2832

Stier A, McKeon C, Osenberg C, Shima J (2010) Guard crabs alleviate deleterious effects of vermetid snails on a branching coral. Coral Reefs 29(4):1019-1022

Tolimieri N (1995) Effects of microhabitat characteristics on the settlement and recruitment of a coral reef fish at two spatial scales. Oecologia 102(1):52-63

Wesner JS, Billman EJ, Belk MC (2012) Multiple predators indirectly alter community assembly across ecological boundaries. Ecology 93(7):1674-1682

White JW, Samhouri JF, Stier AC, Wormald CL, Hamilton SL, Sandin SA (2010) Synthesizing mechanisms of density dependence in reef fishes: behavior, habitat configuration, and observational scale. Ecology 91(7):1949-1961

Wilcox C (2001) Habitat size and isolation affect colonization of seasonal wetlands by predatory aquatic insects. Isr J Zool 47(4):459476

Wilson J, Osenberg CW (2002) Experimental and observational patterns of density-dependent settlement and survival in the marine fish gobiosoma. Oecologia 130(2):205-215

Wratten SD, Gillespie M, Decourtye A, Mader E, Desneux N (2012) Pollinator habitat enhancement: benefits to other ecosystem services. Agric Ecosyst Environ 159:112-122 


\section{A Comparison of the ODE approximation to the mean of the stochastic model}

As described in the Methods section, we use a stochastic and a deterministic model in tandem to study settlement shadow dynamics. Because these mathematical systems are non-linear (by virtue of the density-dependent mortality), the deterministic model is not simply the mean of the stochastic version. In fact, the deterministic model systematically underestimates the mean of the stochastic process due to an application of Jensen's Inequality. We use this subsection to justify this claim.

The stochastic model is a continuous-time Markov chain denoted $N(t)$. For a given initial condition $n \in\{0,1,2 \ldots\}$, we define a collection of functions $\left\{p_{k}(t)\right\}_{k \geq 0}$ that respectively describe the probability that there are $k$ settlers on a focal coral at time $t$,

$$
p_{k}(t)=\mathbb{P}_{n}(N(t)=k)=\mathbb{P}(N(t)=k \mid N(0)=n) .
$$

Recalling that $\gamma$ is the time between settlement pulses, we can write the master equations for this Markov chain for all $t \in[0, \gamma)$ as follows:

$$
\frac{d}{d t} p_{k}(t)=-\left(\alpha k+\beta k^{2}\right) p_{k}(t)+\left(\alpha(k+1)+\beta(k+1)^{2}\right) p_{k+1}(t)+\delta_{0}(t) \sum_{n=0}^{k} \mathbb{P}(\lambda=k-n) p_{n}(0)
$$

The first term on the right-hand side results from the rate of the transition $k \rightarrow k-1$ while the second term corresponds to the transition $k+1 \rightarrow k$. Noting that the size of the initial pulse $\lambda$ is a Poisson distributed random variable, the last term captures the effect of the initial pulse $\left(\delta_{0}(t)\right.$ is a Dirac-delta function concentrated at the initial time $\left.t=0\right)$.

After the initial pulse has occurred, we can look at the derivative of the mean of the stochastic model conditioned on having the initial value $n$, (denoted $\mathbb{E}_{n}[N(t)]$ ),

$$
\begin{aligned}
\frac{d}{d t} \mathbb{E}_{n}[N(t)] & =\frac{d}{d t} \sum_{k=1}^{\infty} k p_{k}(t) \\
& =\sum_{k=1}^{\infty} k\left[-\left(\alpha k+\beta k^{2}\right) p_{k}(t)+\left(\alpha(k+1)+\beta(k+1)^{2}\right) p_{k+1}(t)\right] \\
& =-\sum_{k=1}^{\infty}\left(-\alpha k-\beta k^{2}\right) p_{k}(t) \\
& =-\alpha \mathbb{E}_{n}[N(t)]-\beta \mathbb{E}_{n}\left[N^{2}(t)\right] .
\end{aligned}
$$

By Jensen's Inequality, we observe that

$$
-\alpha \mathbb{E}_{n}[N(t)]-\beta \mathbb{E}_{n}\left[N^{2}(t)\right] \leq-\alpha \mathbb{E}_{n}[N(t)]-\beta\left(\mathbb{E}_{n}[N(t)]\right)^{2}
$$

Now, note that the right-hand side of this inequality is precisely the form seen in the ODE we use in our deterministic approximation $\bar{N}(t)$ defined by Eq. 4 :

$$
\bar{N}(t)^{\prime}=-\alpha \bar{N}(t)-\beta(\bar{N}(t))^{2} .
$$

This implies that the mean of the stochastic process $N(t)$ is decreasing faster than the approximating deterministic process $\bar{N}(t)$. For larger populations, this effect is negligible, but this may not be the case in the dynamics of smaller populations.

\section{B Convergence of the ODE to equilibrium}

As seen in right panel of Figure 1, when the population is relatively large there is a stable recurring pattern in the time periods marked initially by a settlement pulse and followed by a mortality phase. To assign a single number for this pattern, we focus on the sequence of values $\{N(i \Delta)\}_{i=0}^{\infty}$ (Eq. 9) corresponding to the population sizes just before settlement pulses occur. Over time, in the deterministic model, these values converge to a long-term steady value, $\tilde{N}^{*}$. To compute this quantity, we found the solution to the ODE (Eq. 7) between settlement pulses and then note that periodic equilibrium occurs with the number of 
(a)

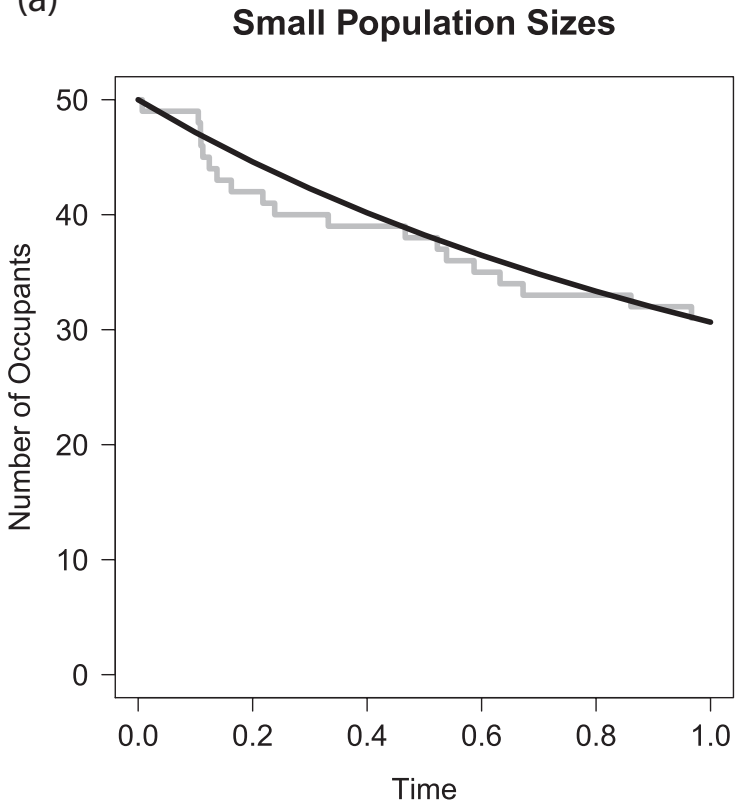

(b)

Large Population Sizes

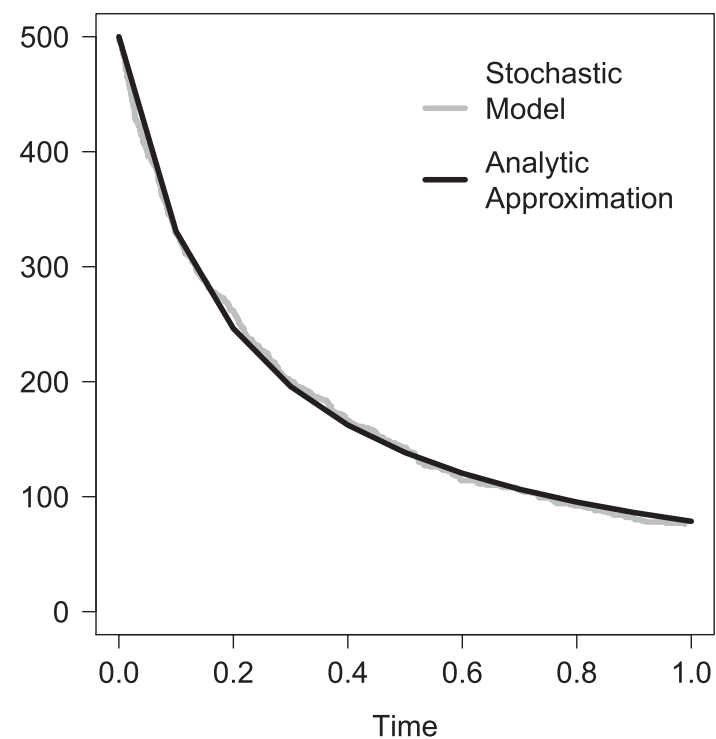

Fig. A1 A comparison of the stochastic model (described in "Post-Settlement Processes") and the analytic approximation for post-settlement mortality (4). Small population sizes started with $\lambda=50$ and large populations with $\lambda=500$. At large population sizes, the analytic approximation nicely approximates the stochastic process. However, at small populations sizes, the approximation underestimates density-dependent mortality.

deaths that occur in the time between settlement pulses is equal to the size of the pulses. That is to say, we look for the point when the sequence of values, $\{N(i \Delta)\}_{i=0}^{\infty}$ (Eq. 9) satisfies the condition $N((i+1) \Delta)=\varphi(N(i \Delta))$ where

$$
\varphi(x)=\frac{(x+v) e^{-\Delta}}{1+(x+v) e^{-\Delta}} .
$$

The function has one fixed point and the formula is given by Eq. 9. Furthermore, because $\varphi$ is increasing and concave down, for all $x<\tilde{N}^{*}$ we have $\varphi(x)>x$. On the other hand, if $x>\tilde{N}^{*}$, then $\varphi(x)<x$. It follows that $\{N(i \Delta)\}_{i=0}^{\infty}$ is a bounded, monotone sequence of points and therefore the sole fixed point is asymptotically stable.

\section{Expected number of settlers on an isolated and a clustered coral}

In our system, colonists arrive as a spatial Poisson process, which is to say that the total number drawn has a Poisson distribution and the location of each is drawn uniformly at random from the domain. The landscape contains circular patches with radius $R$, the centers of which are generated by one of the three spatial point processes (even, random, clustered, described in the main text. Within these landscape configurations, the patches have varying degrees of isolation. The most extreme cases occur when a patch is completely isolated or completely surrounded by other patches. We can analytically compute the exact formula for the distribution of the larval input in these two extreme cases.

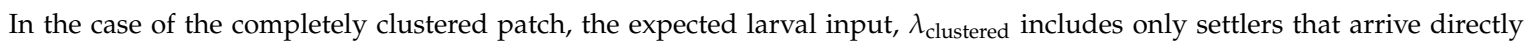
on the coral. Since colonists arrive uniformly at random with density $L$, the mean number of arrivals is simply the density times the area of the patch:

$$
\lambda_{\text {clustered }}=L \pi R^{2}
$$


(a)

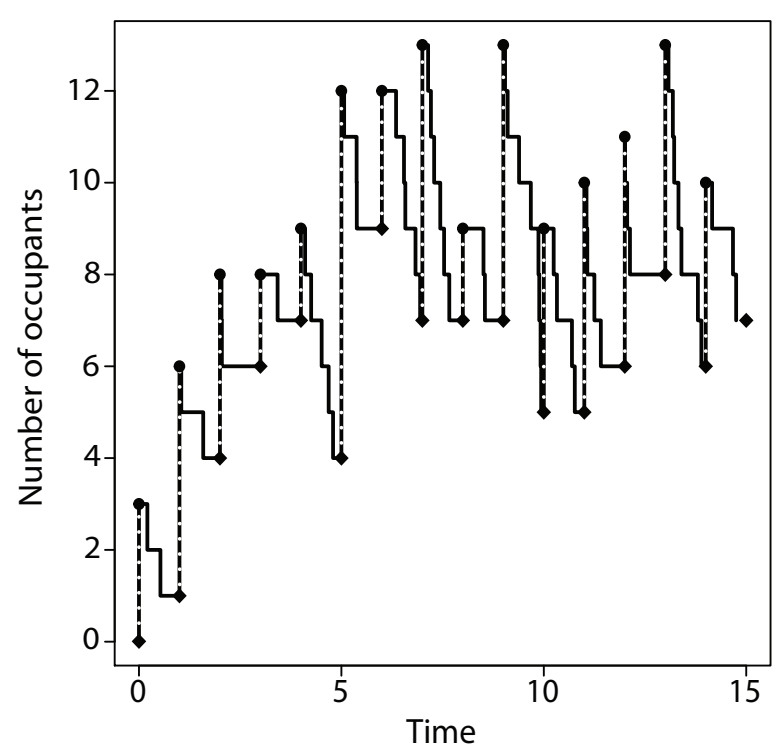

(b) $\lambda=500$

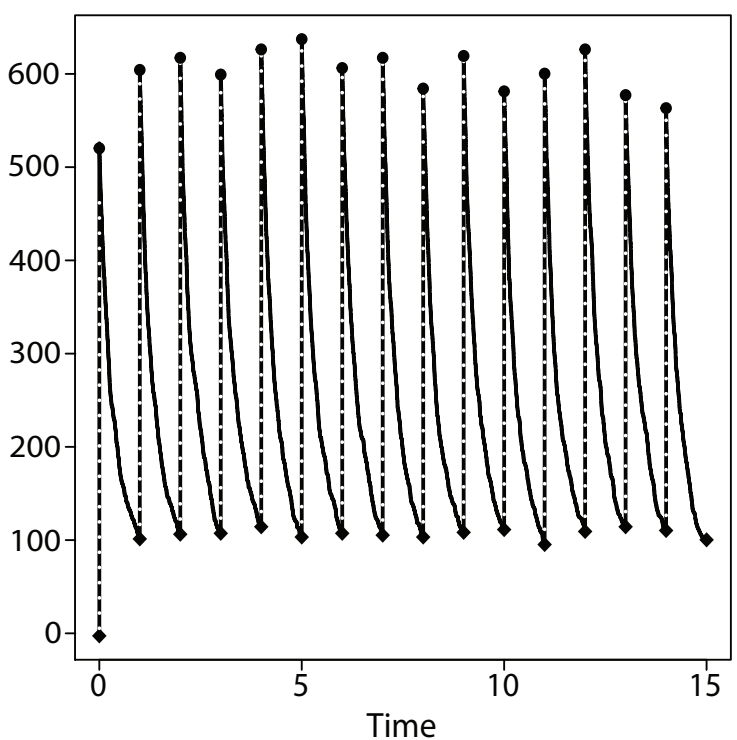

Fig. A2 The stochastic dynamics of fish within a single coral under a) low and b) high settlement intensities. New fish arrive during discrete settlement events (circles) that are followed by continuous (but stochastic) declines in abundance (solid lines) until just before the arrival of another settlement event (diamonds). Empty corals accumulate larvae and eventually converge on an equilibrium at which the number of settlers equals the number of deaths (of recent settlers and older fish) during the interpulse period. At high larval densities, the stochastic dynamics resemble a continuous approximation. In both panels, $\alpha=0.5$, $\beta=0.005$, and $\gamma=1$

Calculating the expected colonist input to a completely isolated coral is more complicated. Suppose there is a circular coral patch of radius $R$ centered at the origin of the $x-y$ plane. We compute the expected number of settlers that arrive at the coral assuming that their initial location in the water column is uniformly distributed over a circular landscape, $\mathcal{O}$, centered at the origin and having radius $R_{\mathcal{O}}$. However, due to the detection threshold, $\theta$, we only consider the area to $R_{*}$, or the radius where the signal is detectable, or where $\theta=m e^{-\rho\left(R_{*}-R\right)}$. Thus, we integrate to $R_{*}=\frac{\ln (m / \theta)}{\rho}+R$.

In this setting, Eq. 3, which describes the probability that any given settler arrives at the focal coral, takes the form

$$
\begin{aligned}
p\left(R_{*}\right) & :=\frac{1}{|\mathcal{O}|} \iint_{\mathcal{O}} \frac{\Lambda_{1}(x, y)}{\sum_{j} \Lambda_{j}(x, y)} e^{-\mu d_{1}(x, y)} d x d y \\
& =\frac{R^{2}}{R_{*}^{2}}+\frac{1}{\pi R_{\mathcal{O}}^{2}} \int_{\theta=0}^{2 \pi} \int_{r=R}^{R_{*}} e^{-\mu(r-R)} r d r d \theta
\end{aligned}
$$

where the first term is the probability that a settler descends into the water column directly onto the coral and the second term includes the possibility of mortality in the travel from the initial location to the coral. Integrating, we find

$$
p\left(R_{*}\right)=\frac{R^{2}}{R_{*}^{2}}+\frac{\mu R+1-\left(\mu R_{*}+1\right) e^{\mu\left(R-R_{*}\right)}}{\mu^{2}}
$$

Substituting $R_{*}=\frac{\ln (m / \theta)}{\rho}+R$,

$$
p\left(R_{*}\right)=\frac{\rho^{2} R^{2}}{\ln (m / \theta)^{2}}+\frac{2 \pi}{\mu^{2}}\left[\mu R+1-\left(\frac{m}{\theta}\right)^{-\mu / \rho}\left(\frac{\mu}{\rho} \ln \left(\frac{m}{\theta}\right)+\mu R+1\right)\right]
$$

The number of settlers that arrive at the focal coral is then a thinned Poisson process with mean $L\left|\pi R_{*}^{2}\right| p\left(R_{*}\right)$

$$
\lambda_{\text {isolated }}=L \pi R^{2}+\frac{2 \pi L}{\mu^{2}}\left[\mu R+1-\left(\frac{m}{\theta}\right)^{-\mu / \rho}\left(\frac{\mu}{\rho} \ln \left(\frac{m}{\theta}\right)+\mu R+1\right)\right] .
$$




\section{Non-dimensionalization}

To non-dimensionalize the ODE (4), we introduce the following rescaled quantities:

$$
\tau=\alpha t, d \tau=\alpha d t, \tilde{N}(\tau)=\bar{N}(t) /(\alpha / \beta) \text {, and } \tilde{\delta}(\tau)=\delta(t) / \alpha
$$

Differentiating $\tilde{N}$ with respect to $\tau$ is related to $d \bar{N} / d t$ by the relations

$$
\frac{d}{d \tau} \tilde{N}(\tau)=\frac{d}{d \tau} \frac{\bar{N}}{\alpha / \beta}=\frac{1}{\alpha} \frac{d}{d t} \frac{\bar{N}}{\alpha / \beta}=\frac{1}{\alpha^{2} / \beta} \frac{d \bar{N}}{d t} .
$$

It follows that

$$
\begin{aligned}
\frac{d \tilde{N}}{d \tau} & =\frac{1}{\alpha^{2} / \beta}\left[\alpha \bar{N}(t)-\beta \bar{N}^{2}(t)+\sum_{k=0}^{\infty} \bar{\lambda} \delta_{k \gamma k}(t)\right] \\
& =\frac{1}{\alpha^{2} / \beta}\left[\left(-\frac{\frac{\alpha}{\beta} \alpha \bar{N}(t)}{\alpha / \beta}\right)-\frac{\frac{\alpha^{2}}{\beta^{2}} \beta \bar{N}^{2}(t)}{\frac{\alpha^{2}}{\beta^{2}}}+\sum_{k=0}^{\infty} \bar{\lambda} \delta_{k \gamma k}(t)\right]
\end{aligned}
$$

If we substitute $\tilde{N}(\tau)=\bar{N} / \alpha / \beta$, then we have

$$
\frac{d \tilde{N}}{d \tau}=-\tilde{N}(\tau)-\tilde{N}^{2}(\tau)+\frac{\bar{\lambda}}{\alpha^{2} / \beta} \sum_{k=0}^{\infty} \delta(t-\gamma k) .
$$

Next, we introduce the non-dimensionlized $\tilde{\delta}(\tau)=\delta(t) / \alpha$

$$
\begin{aligned}
\frac{d \tilde{N}}{d \tau} & =-\tilde{N}(\tau)-\tilde{N}^{2}(\tau)+\frac{\bar{\lambda}}{\alpha^{2} / \beta} \sum_{k=0}^{\infty} \alpha \tilde{\delta}(\alpha(t-\gamma k)) \\
& =-\tilde{N}(\tau)-\tilde{N}^{2}(\tau)+\frac{\bar{\lambda}}{\alpha / \beta} \sum_{k=0}^{\infty} \tilde{\delta}(\alpha t-\alpha \gamma k)
\end{aligned}
$$

Finally, introducing $v=\beta \bar{\lambda} / \alpha$ and $\Delta=\alpha \gamma$, we express the ODE in completely non-dimensional terms:

$$
\frac{d \tilde{N}}{d \tau}=-\tilde{N}(\tau)-\tilde{N}^{2}(\tau)+v \sum_{k=0}^{\infty} \tilde{\delta}_{\Delta k}(\tau) .
$$

\section{E Effect of $\Delta$ on spatial heterogeneity}

The effect of time between settlement pulses relative to the settlement rate depended on $v$. There was a critical value, $v_{\text {isolated }}=$ $\sqrt{\frac{\lambda_{\text {isolated }}}{\lambda_{\text {clustered }}}}$, at which changing $\Delta$ had no effect on the resulting heterogeneity. Instead, at this critical value of $v$, the variation in occupant numbers (between an isolated and clustered coral) is constant and equal to $\sqrt{\frac{\lambda_{\text {isolated }}}{\lambda_{\text {clustered }}}}$ (i.e., heterogeneity was present, but reduced from that imposed at settlement). In addition, as $\Delta \rightarrow 0$ the relative density of occupants approached this same value (i.e., $\sqrt{\frac{\lambda_{\text {isolated }}}{\lambda_{\text {clustered }}}}$; and as $\Delta \rightarrow \infty$, the relative density approached a constant, $\frac{v_{\text {isolated }}\left(1+v_{\text {clustered }}\right)}{v_{\text {clustered }}\left(1+v_{\text {isolated }}\right)}$. Thus, the effects of increased density-independent mortality and time between arrivals (which affect $\Delta$ ) cannot be viewed through the same lens as we viewed effects of $v$. Although increasing $\Delta$ suggests a reduction in saturation, the homogenizing effects of density dependence still operate immediately following settlement pulses. Thus, increasing $\Delta$ cannot strictly maintain the spatial variation created by propagule redirection; some homogenization will be achieved (except in the limiting case as $v \rightarrow 0$ ). 